\title{
Intrinsic Absorption Properties in Active Galaxies Observed with the Far Ultraviolet Spectroscopic Explorer ${ }^{1}$
}

\author{
Jay P. Dunn², D. Michael Crenshaw², S. B. Kraemer ${ }^{3}$, \& M. L. Trippe ${ }^{2}$
}

\begin{abstract}
In a continuing survey of active galactic nuclei observed by the Far Ultraviolet Spectroscopic Explorer, we provide a deeper analysis of intrinsic absorption features found in 35 objects. Our survey is for low-redshift and moderate-luminosity objects, mostly Seyfert galaxies. We find a strong correlation between maximum radial velocity and luminosity. We also examine the relationships between equivalent width (EW), full width at half maximum, velocity and continuum flux. The correlation between velocity and luminosity has been explored previously by Laor \& Brandt, but at a significantly higher redshift and heavily weighted by broad absorption line quasars. We also have examined each object with multiple observations for variability in each of the aforementioned quantities, and have characterized the variation of EW with the continuum flux. In our survey, we find that variability of O VI $\lambda \lambda 1032,1038$ is less common than of the UV doublets of $\mathrm{C} \mathrm{IV}$ and $\mathrm{N} V$ seen at longer wavelengths, because the O VI absorption is usually saturated. Lyman $\beta$ absorption variability is more frequent. In a target-by-target examination we find that broad absorption line and narrow absorption line absorbers are related in terms of maximum outflow velocity and luminosity, and both can be exhibited in similar luminosity objects. We also find one object that shows radial velocity change, seven objects that show equivalent width variability and two objects that show either transverse velocity changes or a change in ionization.
\end{abstract}

Subject headings: galaxies: Seyfert - ultraviolet: galaxies

\footnotetext{
${ }^{1}$ Based on observations made with the NASA-CNES-CSA Far Ultraviolet Spectroscopic Explorer. FUSE is operated for NASA by the Johns Hopkins University under NASA contract NAS5-32985.

${ }^{2}$ Department of Physics and Astronomy, Georgia State University, Atlanta, GA $30303 . \quad$ Email: dunn@chara.gsu.edu,crenshaw@chara.gsu.edu,trippe@chara.gsu.edu

${ }^{3}$ Institute for Astrophysics and Computational Sciences, Department of Physics, The Catholic University of America, Washington, DC 20064; kraemer@yancey.gsfc.nasa.gov.
} 


\section{Introduction}

It is commonly known that intrinsic UV absorption occurs in a large fraction $(\sim 50 \%)$ of Seyfert galaxies. First noticed by Anderson \& Kraft (1969), several surveys have been done in recent years (Crenshaw et al. 1999; Kriss 2002; and Dunn et al. 2007, hereafter Paper I) to characterize these features. In the first step of our survey we examined low-z active galactic nuclei (AGN) (Paper I). Seyfert galaxies are moderately luminous, relatively nearby, and mostly spiral galaxies, which make up a large portion of our survey, while the remainder are low-redshift quasars. We concentrate only on AGN that show broad permitted emission lines and narrow emission lines (i.e., Seyfert 1's, Kachikian \& Weedman 1974). This is because intrinsic absorption is only readily detectable if seen against the broad emission lines and central continuum source characteristic of type 1 objects. Seyfert 2 galaxies have only narrow permitted and forbidden lines, and thus intrinsic absorption cannot be easily detected.

Crenshaw et al. (1999) performed a survey of Seyfert galaxies that had been observed with the Faint Object Spectrograph (FOS) and investigated the properties of the intrinsic absorption lines. They found that nearly $60 \%$ of the targets in the survey showed narrow C IV absorption features (FWHM $<500 \mathrm{~km} \mathrm{~s}^{-1}$ ) and outflow velocities up to $2000 \mathrm{~km}$ $\mathrm{s}^{-1}$. Also in this survey, they found a link between warm X-Ray absorbers and UV intrinsic absorption, in the sense that Seyfert galaxies that show one also show the other.

Broad absorption line (BAL) quasars have been studied even more extensively. These objects show lines with much larger velocity widths, up to $\sim 10,000 \mathrm{~km} \mathrm{~s}^{-1}$ (Weymann et al. 1981, Turnshek 1988), which appear in $\sim 10 \%$ of all quasars (Foltz et al. 1990). This is a much smaller percentage than the percentage that exhibit narrow absorption lines only, so the global covering factor is an important consideration in determining their nature.

Laor \& Brandt (2002) looked for various trends concerning intrinsic absorption in data from the Hubble Space Telescope (HST) and the International Ultraviolet Explorer (IUE). Their survey was derived from the PG quasar sample (Boroson \& Green 1992). One of the more notable trends they found was a dependence of maximum outflow velocity on luminosity

$\left(\mathrm{v}_{\max } \propto \mathrm{L}^{0.62 \pm 0.08}\right)$. Their plot contains both BAL and narrow absorption lines (NALs). They found that the equivalent width (EW) also followed an increase with increasing luminosity. This trend is based on Soft X-Ray Weak Quasars (SXWQs), which show larger values of EW and $\mathrm{v}_{\max }$ than any other quasar across the luminosity span. They furthermore showed that [O III] EW and $\mathrm{v}_{\max }$ are correlated. SXWQs showed a significant correlation of EW versus $\mathrm{v}_{\max }$. Of the 56 objects they investigated, 28 showed intrinsic UV absorption. This agrees well with the $\sim 60 \%$ frequency Crenshaw et al. (1999) found in their lower-luminosity sample and the $\sim 50 \%$ frequency we found in Paper I. 
For a purely BAL sample, Ganguly et al. (2007) recently used data from the Sloan Digital Sky Survey (SDSS) to investigate their properties. They conclude that the overall fit between $\mathrm{v}_{\max }$ and luminosity found by Laor \& Brandt applies to their much larger sample of over 5000 objects. In Ganguly \& Brotherton (2008), they compile all intrinsic absorption features for all known surveys and show that the fit still holds.

Our knowledge of the variability for intrinsic absorption is limited. Variability is important because it is our only link to several key physical parameters concerning the AGN. Cromwell \& Weymann (1970) were the first to detect variability, in the Seyfert 1 NGC 4151. Later, in data from the $I U E$, more absorption variability was seen (Crenshaw et al. 1999). Nearly all of the variability has been due to changes in the ionic column densities, as a result of changes in the ionizing flux from the continuum source. However, in four Seyfert galaxies (NGC 3516, NGC 3783, NGC 4151, and NGC 5548), bulk transverse motion of gas across the line of sight was identified as a likely culprit (Crenshaw et al. 2003). In the bright Seyfert galaxy NGC 4151, both ionization change and bulk transverse motion were detected. Kraemer et al. (2006) performed a detailed analysis of the absorption from the Space Telescope Imaging Spectrograph (STIS) data. For the component they dubbed D+Ed, they found a transverse velocity lower limit of $\mathrm{v}_{t} \geq 2100 \mathrm{~km} \mathrm{~s}^{-1}$.

NGC 3783 has shown previously to be variable (Gabel et al. 2005, Maran et al. 1996). In 1993 February data from STIS, NGC 3783 showed a spectrum with no narrow intrinsic absorption. By 1994 January, two obvious absorption features arose for each member of the C IV doublet. By 2000 February another pair of absorption lines had emerged. Gabel et al. (2003) showed that the radial velocity changed as well. In a series of several spectra the radial velocity of the line changed in two time spans. They first found a change of $\sim 35 \mathrm{~km} \mathrm{~s}^{-1}$ and in the second span they saw a change of $\sim 55 \mathrm{~km} \mathrm{~s}^{-1}$. This is the first well-documented case of a change in radial velocity for an intrinsic absorber in a Seyfert galaxy. Transverse velocity was also measurable, where they found a lower limit of $\mathrm{v}_{t} \geq 540 \mathrm{~km} \mathrm{~s}^{-1}$, which is comparable to the radial velocity $\left(\sim-450 \mathrm{~km} \mathrm{~s}^{-1}\right)$. Motivated by their findings, we undertook a survey of O VI and Ly $\beta$ absorption in FarUltravioletSepctroscopicExplorer (FUSE) spectra of low-redshift AGN.

The penultimate goal of our research is to understand the general dynamics of mass outflow and to ultimately help complete the picture of the AGN central engine. Several dynamical scenarios have been offered as explanations for the outflow (see Crenshaw et al. 2003 for a review of the models). One method, Compton heated winds assumes that EUV and X-ray photons from the accretion disk are irradiating the gas at larger distances and creating a thermal wind (Begelman et al. 1983). Radiative driving is also a promising method to explain fast-moving outflows such as BALs (deKool \& Begelman 1995, Arav et 
al. 1994). Another possibility is that magnetic fields from the accretion disk are lifting plasma from the disk in magnetohydromagnetic (MHD) flows, where the plasma follows a helical magnetic field line (Blandford \& Payne 1982). Depending on how far from the central core these clouds can follow the field lines, this could also be an explanation for the high transverse velocities.

In Paper I we cataloged 35 AGN with intrinsic absorption and presented approximate velocities. We found 11 new UV intrinsic absorption objects in a sample of 90 nearby AGN observed by FUSE. We calculated the frequency of O VI absorption to be on the order of $50 \%$, slightly lower than the value Crenshaw et al. (1999) found for C IV. The global covering factor, based on an estimate of the average covering factor in the line of sight $\left(\left\langle\mathrm{C}_{f}\right\rangle\right)$ was similar to previous results of $\sim 0.4$. We continue our study of that absorption measurements of the absorption lines we found in the 35 objects from Paper I.

\section{Optical Observations and Measurements}

We obtained ground-based optical spectra to obtain redshifts and/or estimate blackhole masses and to supplement the FUSE data for three objects: IRAS F22456-5125, MR 2251-178 and WPVS 007. These data are readily available in the literature for most of the other objects. Each galaxy was observed in both the blue and red optical regions with the R-C spectrograph on the Cerro Tololo Inter-American Observatory (CTIO) 1.5-m telescope in Chile. Table 1 chronicles the dates, integration times and wavelength coverage for these data. The blue spectra (spanning from approximately 3360 to $5440 \AA$ ) were taken using a grating with a dispersion of $\sim 1.47 \AA$ pixel $^{-1}$, providing a resolution of $4.3 \AA$. The red spectra (5652 to $6972 \AA$ ) were taken using a Schott GG495 filter and a grating with a 1.10 Apixel $^{-1}$ dispersion grating giving us a resolution of $3.1 \AA$. All objects were observed through a long slit with a width of $4^{\prime \prime}$. The stars LTT 4364 and Feige 110 were observed with the same settings for the purpose of flux calibration. The spectra were reduced and calibrated using standard IRAF reduction packages for long-slit spectroscopy. We show the reduced spectra in Figure 1.

For the source IRAS F22456-5125, which had a redshift estimate listed on NASA/IPAC Extragalactic Database (NED) of 0.1 (Mason et al. 1995), we used our spectra to estimate the redshift based on the positions of lines in the blue spectrum. The centroids of the emission lines [O II] $\lambda 3727,[\mathrm{Ne} \mathrm{III}] \lambda 3869,[\mathrm{Ne} \mathrm{V}] \lambda 3424$ and $\mathrm{H} \beta$ were available in the wavelength

coverage of the blue setting and we found an average redshift of $z=0.1016 \pm 0.0001$. The redshifts from NED for WPVS 007 and MR-2251-178 are consistent with our measurements. 
The optical spectra allowed us to determine mass estimates for the central supermassive black hole $(\mathrm{SMBH})$ of these objects via the empirical relationships between SMBH mass and the $\mathrm{H} \beta$ FWHM and continuum luminosity at $\lambda=5100 \AA$ (or, equivalently the $\mathrm{H} \beta$ line luminosity) calibrated by Vestergaard and Peterson (2006) using emission-line reverberation mapping. We present these masses along with the masses of the rest of the sample later in this paper.

\section{UV Observations and Measurement}

We have 105 observations from FUSE of the 35 targets found with intrinsic absorption from Paper I (see Table 1 from Paper I). FUSE consists of four telescopes each with four gratings and two detectors with a spectral resolution of $\sim 15 \mathrm{~km} \mathrm{~s}^{-1}$, which provides 8 spectra per observation covering the wavelength range of $905 \AA$ to $1187 \AA$. The detectors have two different coatings, $\mathrm{LiF}$ and $\mathrm{SiC}$. The detector with the $\mathrm{LiF}$ coating provides a (nearly twice) higher reflectivity than the $\mathrm{SiC}$ coating (Sahnow 2002). The $\mathrm{SiC}$ and Lif detectors do have overlapping regions that allow for the spectra to be coadded, weighted by exposure time, and scaled to the LiF 1a spectrum. We downloaded the spectra from MAST and used CalFUSE 3.1 to process the raw data in time-tag mode (Dixon et al. 2002). Further details of the reduction process can be found in Paper $\mathrm{I}$.

\subsection{Light Curves}

Generating a continuum light curve for FUSE data proved to be a difficult task due to the nature of the two detectors and redshifted broad emission lines. Using the same method as described by Dunn et al. (2006), we chose $1110 \AA$ in the observed frame as a good position to measure the continuum flux. In a $10 \AA$ bin, both detectors contribute to the measured flux at that wavelength. In some spectra from either the $\mathrm{LiF}$ detector or the $\mathrm{SiC}$ detector, the target was sometimes out of the aperture. In these cases we did not include the segments. Also, due to redshift considerations, we were forced to move the bin when the redshift was $\geq 0.6$. At those particular redshifts the broad line would provide a measured flux value too high for the continuum by up to 20-30\%. For the majority of the observations, the flux we provide is an average flux within the $10 \AA$ bin at $1110 \AA$; this avoids interstellar medium (ISM) lines (Morton 1991) and geocoronal dayglow lines (Feldman et al. 2001). The rest of the observations were measured in a $20 \AA$ bin taken at 1160 or $1020 \AA$, depending on which was closest to and unobstructed by the O VI broad line. We converted our fluxes to log specific luminosity, and provide flux, log luminosity, bin position, and errors in Table 2. 


\subsection{Measured Absorption Quantities}

We measured the EW, full width at half-maximum (FWHM) and radial velocity centroid with respect to systemic redshift of each intrinsic absorption feature identified in Paper I. These measurements are listed in Table 3 for the Ly $\beta$, O VI $\lambda 1032(\mathrm{O}$ VIb) and O VI $\lambda$ 1038 (O VIr) lines. Many features were blended or too contaminated by galactic absorption features to be measured accurately, thus they have been excluded. Because FUSE has no onboard calibration lamp, CalFUSE is designed to apply a fit to the spectrum based on a predetermined wavelength solution (Sahnow 2000). This can lead to an inaccuracy of up to $20 \mathrm{~km} \mathrm{~s}^{-1}$ (Gillmon et al. 2006). We measured available ISM lines near our intrinsic absorption features, in order to avoid nonlinear calibration effects, to correct the velocities. These corrections have already been accounted for in our velocities in Table 3.

Errors for EWs were based upon the signal-to-noise $(\mathrm{S} / \mathrm{N})$ ratio of the data along with the standard deviation of three measurements of each line with three different continuum estimates. The quoted error is a sum of these two values. Because velocity centroids, which are depth weighted, have small standard deviation errors for one line, we used the mean standard deviation for the three lines ( $\mathrm{Ly} \beta, \mathrm{O}$ VIb, O VIr) in each observation. For FWHM we estimated the error as the standard deviation of the three measurements per line, similar to EW.

We estimated flux error from the standard deviation within the 10 or $20 \AA$ bin. This is typically an overestimate of the error due to oversampling, similar to the case for IUE data (Dunn et al. 2006). We find that we overestimate by nearly a factor of 2.5 by examining spectra close in time $(\lesssim 1$ day) for like objects. Because most of these objects are Seyfert galaxies, the flux level is not likely to change significantly in the span of $\lesssim 1$ day. We therefore scaled our flux errors by this amount.

Our listed redshifts for the objects are provided by NED. We chose them based on the recommended most accurate value, preferably from HI-21 cm measurements. However, not

all objects have very accurate values for the redshift, such as IRASF 22456-5125 ( $\oint 2$ ). Some of the redshifts are from narrow emission lines, which are often blue shifted with respect to HI-21 cm values (Crenshaw et al. 1999). This may explain the slightly positive radial velocities found for some components in a few objects.

In several cases we found that of the three lines we examined, only one or two of the lines were measurable. This is due to either poor signal-to-noise, Ly $\beta$ being too weak, or heavy blending with ISM features. 


\section{Correlations}

All of our correlations involving EW, FWHM and/or luminosity show measurements from each absorption component in each observation, because many of these quantities can be variable over time. In order to inspect our plots for correlations we simply examine by eye the data for either a direct trend or for an envelope fit to the data, as in Laor \& Brandt (2002).

The plots containing velocity measurements are averaged over time due to the lack of significant variability in velocity space. Also, error bars for many of the points are contained within the plot symbol. The most tantalizing correlation we have found is that of velocity with luminosity. While this was seen in C IV by Laor \& Brandt (2002), their objects were mostly quasars with high luminosities and redshifts beyond 1.5, which was our redshift cutoff on the high end. We share four objects in common. We plot in Figure 2 all of our velocities measured for each object and each component. In general as the luminosity increases, it appears that the maximum velocity at each luminosity does as well. This confirms the trend that Laor \& Brandt found for higher-luminosity objects and shows that the trend also holds for lower-luminosity objects such as Seyfert galaxies. To confirm this further, we converted the magnitudes provided by Laor \& Brandt to $\log \mathrm{L}_{\lambda}$ by using the four targets we share and found a scale factor of 1.7. We plot the Laor \& Brandt observations along with our own measurements in Figure 3 and see that the two data sets follow remarkably similar trends. The relation given by Laor \& Brandt is $\mathrm{v}_{\max } \propto \mathrm{L}^{0.62 \pm 0.08}$. While we do not calculate this, we can see by Figure 3 that all of our points fall beneath this maximum velocity trend.

Ganguly \& Brotherton (2008) show a similar plot. They have plotted the Laor \& Brandt (2002) data along with their SDSS data from Ganguly et al. (2007), and data from several other sources (references within). The plot shows that for all available data the trend from Laor \& Brandt holds for all luminosities and velocities.

Along with the measured black hole-masses from the optical data, we compiled all of the known masses for our survey objects in Table 4 (we exclude those few without the necessary measured quantities) and calculated the Eddington ratio. For all objects referencing Peterson

et al. (2004), the masses are from reverberation mapping; all others were calculated using the relationship found by Kaspi et al. 2000 and calibrated by Vestergaard \& Peterson (2006):

$$
M_{B H}=\log \left[\left(\frac{F W H M(H \beta)}{1000 \text { km s }^{-1}}\right)^{2}\left(\frac{\lambda L_{5100}}{10^{43} \text { ergs s }^{-1}}\right)^{0.5}\right]
$$

using the FWHM of the broad $\mathrm{H} \beta$ emission line and the luminosity at $5100 \AA$. We find our value for $\mathrm{L}_{B o} l=9.8 \lambda \mathrm{L}_{5100}$ from McLure \& Dunlop (2004). 
We plot the maximum velocity, as before with luminosity, against the Eddington ratio $\left(\mathrm{L} / \mathrm{L}_{E d d}\right)$ in Figure 4. There appears to be a weak trend of maximum velocity the with Eddington ratio. It should be noted that the two objects with the highest velocities have low ratios, and the two objects with a high ratio have low velocities. In order to complete the picture we would require more data and a wider range of objects spanning the range of ratios as most of our targets are moderate Eddington accreting objects.

We examined how the EW relates to the specific luminosity in our sample, which we show in Figure 5. Overall there appears to be no trend. This contradicts the findings of Laor \& Brandt (2002) for C IV lines, but this could be a simple case of saturation affecting our EW measurements. The only notable feature in the plot is the lack of points for low luminosity and low EW. The paucity of points is most likely a selection effect of low signalto-noise for weak lines in objects with low-continuum fluxes. It should be noted that, some of our measured EWs are measured over what have been seen, in less saturated UV absorption lines, to be multiple components (e.g., NGC 4151 in Kraemer et al. 2005). Due to the heavy blending and saturation in O VI, we simply measure across blends as one component. We have removed any measurements of lines in spectra with a luminosity signal-to-noise of $<30$ and measurements from spectra that are saturated with multiple components, and still find that there is no trend.

We additionally investigated the relation between EW and maximum velocity (not shown). Again, there appears to be very little trend. We also examined the relation between FWHM and the specific luminosity, as shown in Figure 6. There appears to be no relation between FWHM and luminosity. However, as seen with EW, there does appear to be a lack of absorption for O VI at low luminosities and small FWHMs.

\section{Variability}

Our survey is the first to date with a significant number of objects with multiple spectra to provide enough data for a time series analysis of intrinsic absorption variability in the far ultraviolet. Of the 35 targets found in the Paper I survey, 22 of the objects have multiple observations. Using the EWs, velocities and FWHMs we provided in Table 3, we examined a time series of each along with the corresponding fluxes for each observation from Table 2. Along with the times series data, we plotted the spectra for each object in chronological order to see visually how the absorption features changed. While most objects showed no real trends between EW, FWHM, or velocity vs. continuum flux, we found a handful of cases that do. 
Mrk 79 is one case of a likely variation. $\operatorname{Ly} \beta$ is clearly visible in the observations in Figure 7. Component 1 of $\mathrm{O}$ VIb is a broad feature due to blending with ISM lines. O VIr is a narrow, most likely saturated line. The variability for Mrk 79 is seen in the O VIr line. O VIr changes in EW for component 1, but does not appear to be correlated with continuum luminosity in Figure 8.

NGC 4151 has broad, saturated absorption troughs. These troughs are comprised of several blended components seen as individual features in STIS data (Kraemer et al. 2001). Because they are blended, it makes measuring individual EWs, FWHMs and velocities difficult. One interesting point is that in the first two observations, seen in Figure 9, the broad trough shows some structure, but in the subsequent observations, they blend too much to discern any one particular absorber. As shown in Kraemer et al. (2001), one particular component, labeled $\mathrm{D}^{\prime}$, only appears in weak flux states. In Table 2 we see that the earlier observations are weaker in flux, and that the $\mathrm{D}^{\prime}$ component flattens the region between $\sim 1029$ and $1032 \AA$, which is consistent with the interpretation of Kraemer et al.

Mrk 817 is another object that shows evidence of variability. One of the labels in Dunn et al. (2007) is incorrect; the Lyman $\beta$ line is weak, and the line marked component 1 at $1064 \AA$ is $\mathrm{H}_{2}$. In Figure 10, we see that Mrk 817 shows in O VIb that component 1 evolves from two subcomponents to a smoother single component. It is possible that these subcomponents are hidden within the artificially broadened O VIr line due to galactic Fe II $\lambda$ 1055. Lyman $\beta$ is not visible most likely due to high ionization. This is confirmed by STIS spectra that show weak absorption in Lyman $\alpha$ (Penton, Stocke \& Shull 2002). The EW for the original lines shows a correlation with the decreasing continuum flux (Figure 11), and FWHM shows the same. While this is a $2 \sigma$ detection in $\mathrm{O}$ VIb, the similar behavior of all three lines leads us to believe that the change is real. The velocity seems to drop for Mrk 817 showing a significant decrease between the first observation and the last. All of this suggests two blended components in the original observation, and that the higher-velocity component decreased with time.

Mrk 817 also demonstrates the appearance of a previously unseen line. At $\sim 1052 \AA$ and $\sim 1058 \AA$ we see that in the first spectrum there is no significant sign of an absorption feature. However in the third spectrum there appears to be a slightly depressed broad feature, and in the final spectrum the broad line is clear with two O VI features on either side at the appropriate locations in velocity space $\mathrm{v}_{r}=3700 \& 3430 \mathrm{~km} \mathrm{~s}^{-1}$. However, we cannot rule out the possibility that the new component is present in the previous observations but not detectable due to the lower $\mathrm{S} / \mathrm{N}$.

In Figure 12, we present the available spectra for Mrk 279. Figure 13 shows the relation of EW and continuum flux for Mrk 279, which shows a weak trend. In both O VI lines 
the EW increases with decreasing flux. For Ly $\beta$ the relationship with flux is much flatter. During the last three observations we see the flux drop by $\sim 60 \%$ and then increase by $\sim 50 \%$, while the EW for all three lines shows a chronological decrease. We see that O VIb varies more than O VIr in Mrk 279. This is most likely due to contamination in both of the O VI lines from ISM and $\mathrm{H}_{2}$. While these lines are heavily contaminated (see Paper I), we can still measure variation as the ISM lines should experience no change.

Mrk 290 (Figure 14) provides a case for a weak anti-correlation between EW and flux. In this object the EWs for both members of the O VI doublet $\lambda \lambda 1032$ and 1038 lines show a slight decrease with a significant increase in continuum flux over a 1200 day interval, and a moderate decrease in the Ly $\beta$ width, as shown in Figure 15.

The variability in Mrk 509 was complex, as shown in Figure 16. What we measured for component 1 , can be seen in Lyman $\beta$ to be at least two subcomponents in two of our three spectra. What we call component 2 can be seen in Lyman $\beta$ to be comprised of at least two or three subcomponents. Kraemer et al. (2003) confirm this by identifying 8 components in spectra from FOS and STIS, which are blended together with the two main components we see in the FUSE data. The components labeled 1-3 by Kraemer et al. are subcomponents that are contained in our measured component 1, while those labeled 4-8 by Kraemer et al. make up our measured component 2. Subcomponents 3 and 6 in Lyman $\beta$, labeled by Kraemer et al. appear to vary. Subcomponent 3 shows an decrease in depth while subcomponent 6 increases in depth over time. We discuss the depth further in the next section. Our measured component 1 shows no change in EW or velocity over time (Figure 17), but our measured component 2 shows a slight increase in Ly $\beta$ (Figure 18).

Figure 19 shows the spectra of PG 0804+761, which exhibits evidence of radial velocity change. This change is seen in all three lines as a centroid shift to more negative velocities, while the ISM lines remain stationary. We plot these centroid velocities vs. time (Figure 20). PG $0804+761$ has a poor estimate of redshift, similar to IRAS F22456-5125. Thus the absolute values of velocity reflect redshifted lines, but this might change with a more accurate redshift.

In the object NGC 3783, we find variability of EW in the Ly $\beta$ line. Figure 21 shows the time evolution of the spectrum, and Figure 22 shows the relation between flux and EW and velocity over time. The EWs for O VI $\lambda 1038$ and O VI $\lambda 1032$ show no correlation, across the dashed average EW line. Ly $\beta$ decreases from low flux to high flux. However, this is evidently a blended combination of two lines labeled 2 and 3 in a paper by Gabel et al. (2003). Thus it is difficult to determine if one or both lines are responding to continuum change. In Figure 21, we see that components 1 and 4 from Gabel et al. (2003) are visible in Ly $\beta$, and are hard to discern in O VI $\lambda 1032$ due to heavy contamination from C II and 
$\mathrm{H}_{2}$. For $\mathrm{O}$ VI $\lambda 1038$ there are two $\mathrm{H}_{2}$ lines nearby, but the weak component 4 is not visible.

\section{Discussion}

In the Kraemer et al. (2005, 2006) study of the variability in NGC 4151, they found some cases where the EWs of lines were anti-correlated with the continuum flux level, while others showed no relation to the flux. We see similar events in only a few objects such as Mrk 817, where the O VI lines seem to follow the continuum, and Mrk 79, where the EWs vary but the continuum stays relatively constant. The paucity of $\mathrm{O}$ VI variability suggests that O VI, which is usually predicted to be very strong in absorber models, is saturated in most cases. It should also be noted that in many cases there is a lack of change in Ly $\beta$, this is presumably also due to saturation. The changes we do see in EW must be due to one of the three events: a covering factor change in the line of sight, a change in ionization, or a total column change (characterized by $\mathrm{N}_{H}$ ) due for example, to bulk motion of gas into our line of sight.

\subsection{Potential Cases of Bulk Motion}

There are three targets in our survey that show changes in the absorption are the best candidates for bulk motion of gas across the line of sight with a transverse velocity $\left(\mathrm{v}_{t}\right)$, as described below. These three objects are Mrk 79, Mrk 817 and Mrk 509.

Mrk 79 is an interesting target because while the EW of component 1 is variable, the continuum does not change. This lends itself to a case of changing the total column, most likely due to bulk motion. We can calculate the transverse velocity by using the measured broad line diameter from Peterson et al. (1998) of 36 light days and a time interval between observations $(\Delta \mathrm{t})$ of 83.7 days the absorber would be moving at a transverse velocity $\geq 126,000 \mathrm{~km} \mathrm{~s}^{-1}$. Given the average radial velocity of the absorber $\mathrm{v}_{r} \approx 300 \mathrm{~km} \mathrm{~s}^{-1}$, the estimate for the transverse velocity is inordinately high. We will tackle further explanations in the next section.

In the case of Mrk 817, if we assume that the appearance of the new absorption components (2 and 3 ) are due to transverse motion of material into the line of sight, we can follow the methods used by Gabel et al. (2005) for NGC 3783. Assuming the O VI doublet is not

saturated we can use the covering factor in the line of sight for $\mathrm{O}$ VI at the conclusion of 
the variability event with the equation:

$$
C_{f}=\frac{I_{1}^{2}-2 I_{1}+1}{I_{2}-2 I_{1}+1}
$$

(Crenshaw et al. 2003), where $I_{1}$ is the intensity at the core of the stronger oscillator strength line and $\mathrm{I}_{2}$ is for the weaker line. This number can be skewed an by additional flux from the Narrow Emission Line Region (NELR) (Kraemer et al. 2002, Arav et al. 2002, Crenshaw et al. 2003), but for Mrk 817 the narrow emission lines are not prominent, and we find $\mathrm{C}_{f}=$ 0.70. If we compare this to the line-of-sight covering factor of 0.51 and 0.33 for the blue and red lines respectively we verify that the lines are not saturated due to the fact that as lines approach saturation in a doublet, the line-of-sight covering factor approaches the doublet covering factor. The transverse size of the absorbing cloud is $\mathrm{d}=\mathrm{d}_{B L R} \times \sqrt{C_{f}}$ (Crenshaw et al. 2003). For Mrk 817, the BLR is 31 light-days across (Peterson et al. 1998). The absorption appeared over $\sim 310$ days, which gives a lower limit to the transverse velocity of $\sim 25,100 \mathrm{~km} \mathrm{~s}^{-1}$. This is exceptionally large compared to the radial velocity of 3700 and 3430 $\mathrm{km} \mathrm{s}^{-1}$ for the two new components. Thus, either the components were buried in the noise in the earlier observations or we are witnessing their appearance due to ionization changes.

Mrk 509 was shown in Peterson et al. (1998) to have a BLR size of 159 light days. If we assume that subcomponents 3 and 6 are saturated in $\mathrm{Ly} \beta$, then we can calculate the change in covering factor $\left(\mathrm{C}_{f}\right)$ from the residual intensities in the cores $\left(\mathrm{C}_{f}=1-\mathrm{I}_{r}\right)$. In the case of subcomponent 6 , we measured the core and found average covering factors $\mathrm{C}_{f}=0.78$ and 0.96 for observations 1 and $3(\Delta t=307.3$ days). For subcomponent 3 , we find covering factors $\mathrm{C}_{f}=0.93$ and 0.77 for observations 2 and $3(\Delta \mathrm{t}=304.0$ days). Subcomponent 6 therefore, showed an increase in covering factor by 0.18 while subcomponent 3 showed a decrease by 0.16. Based on the changes in covering factors, from our previous equation we can find lower limits for transverse velocity for these of $65,600 \mathrm{~km} \mathrm{~s}^{-1}$ for subcomponent 6 and 125,200 km $\mathrm{s}^{-1}$ for subcomponent 3. This seems extremely high for a transverse velocity when compared to the radial velocities of $\sim 300 \mathrm{~km} \mathrm{~s}^{-1}$. Therefore, the changes in the depths of these two subcomponents are almost certainly due to ionic column variation and not covering factor changes.

Thus, even in the most favorable cases, we find no evidence for EW changes due to bulk motion. Because there is no evidence that transverse velocities of UV absorbers can reach these inordinately high values (Crenshaw et al. 2003), the only viable alternative is ionic column changes due to changes in the ionizing continuum. 


\subsection{Ionization Changes}

The three objects we have ruled out bulk motion for and the other six objects that showed signs of absorption variability all seem to be the result of changes in the ionizing flux. Mrk 279 and Mrk 290 showed an anticorrelation between ionization parameter (U) and O VI EW, while NGC 3783 showed anticorrelation in Ly $\beta$. For Mrk 279 and Mrk 290 this means that the ionization state is high, and NGC 3783 is indeterminate due to no detectable change in O VI. Mrk 817, component 1, is the only case where the EW for O VI is correlated with the flux, which means that $\mathrm{U}$ is relatively low. For NGC 4151 we see the $\mathrm{D}^{\prime}$ component decrease with increasing flux, thus it is another high-U component. PG 0804+764 showed correlation between flux and EW for both the O VIb line and Ly $\beta$. This object stands out because it is the only object in the survey that showed an unexplainable change in radial velocity.

Mrk 79 showed a change in EW and no change in flux. Because we only have three observations, we believe what we are seeing a change in ionization with a time delay. Our observations do not accurately sample the lightcurve and there must be a delay between the EW response and the continuum, due to low densities and/or large distances of the absorbers from the continuum source (Crenshaw et al. 2003).

The appearance of the absorption components 2 and 3 in Mrk 817 is most likely due to a change in ionization. The light curve shown at the bottom of Fig. 11, shows that in the last observation where the two components are visible, the flux level had decreased significantly. The appearance of these lines may therefore be due to a decrease in ionizing flux. We can also use the possible disappearing subcomponent in component 1 to place limitations on the absorption systems. As the continuum flux decreases the line weakens and vanishes/blends with the component at $\sim 1049.2 \AA$, and could be the explanation of the EW variations we noted in $\oint 5$. In order to place these constraints, we need CLOUDY modeling of the absorbers.

Mrk 279 presents a similar situation. In Figure 13 we have a range of $\sim 58$ days where the continuum flux drops and rises. The EW for the absorbers respond by decreasing. We could estimate a lower limit to the distance and density provided a model of the absorber and assuming the response is complete after 58 days. Ideally, we would need a monitoring campaign with several observations for an event such as this to fully explain the variations.

For the component Kraemer et al. (2002) labeled 3 in Mrk 509, we can assume that the ionizing flux increased and the column changed. We know from Kraemer et al. (2003) that the ionization parameter (U) for Mrk 509 in subcomponent 3 is 0.03 . Given the change in 
time, $\Delta \mathrm{t}=2.6 \times 10^{7} \mathrm{~s}$, we can use the equation as seen in Krolik \& Kriss (1995):

$$
t_{i o n}=\frac{h \nu}{F_{\text {ion }}<\sigma_{\text {ion }}>}
$$

where $\nu$ is the transition frequency, $\mathrm{F}_{i o n}$ is the ionizing flux at the cloud front and $\left\langle\sigma_{\text {ion }}\right\rangle$ is the ionization cross section (Osterbrock 1989). Using our known limit on the timescale, and a calculated value of $\mathrm{L}_{i o n}=2.1 \times 10^{44} \mathrm{ergs} \mathrm{s}-1$, we can get a limit on the distance from the source to the cloud from $\mathrm{F}_{i o n}=\mathrm{L}_{i o n} / 4 \pi \mathrm{d}^{2}$. That gives us the lower limit to the distance of $14 \mathrm{pc}$.

Finally, we can estimate the density of the absorbing cloud assuming a change in ionization, from the ionization parameter:

$$
U=\frac{\int \frac{L_{\nu} d \nu}{h \nu}}{4 \pi c r^{2} n_{e}}
$$

For our subcomponent 3 we find $\mathrm{n}_{e} \leq 1.38 \times 10^{3} \mathrm{~cm}^{-3}$.

\subsection{NALs and BALs}

From both the Paper I survey and the Laor \& Brandt surveys, one object, PG 1411+442, appeared in both surveys with BAL and NAL signatures. The NALs appear superimposed on the BAL in both members of the O VI doublet, as seen in Paper I. This evidence tied with the Laor \& Brandt and our relationship from section $\oint 4$, shows that both BALs and NALs are related and follow the same trends and that they both may exist simultaneously in an object.

Also from our previous survey, WPVS 007 was omitted from the search for NALs due to the discovery of a newly appearing BAL by Leighly et al. (2007, in prep). We have reexamined this object and found that the NALs seen by Crenshaw et al. (1999) are still visible in the FUSE spectrum. The NALs for O VI and Ly $\beta$ are buried in the BAL and have a velocity of $-399 \pm 10 \mathrm{~km} \mathrm{~s}^{-1}$ seen in Figure 23. This matches the velocity Crenshaw et al. (1999) found of $-390 \mathrm{~km} \mathrm{~s}^{-1}$.

We can evaluate the configuration of the NAL and the appearing BAL in WPVS 007. The time difference between the FUSE spectrum and the last FOS spectrum is 2655 days. If we use the relationship from Vestergaard \& Peterson (2006):

$$
R \propto L_{\nu}(\lambda 5100)^{0.50 \pm 0.06},
$$

with a BLR size that has been estimated from reverberation mapping, we find that the BLR size for WPVS 007 is 23 light days across. This gives a lower limit on the transverse velocity 
of the BAL of $2565 \mathrm{~km} \mathrm{~s}^{-1}$. The NAL however is still apparent in the O VI doublet and Lyman $\beta$ lines. This leaves us with two situations. Either the NAL is interior to the BAL and is still receiving sufficient ionizing flux to maintain the absorption, or the NAL is exterior to the BAL and is no longer receiving ionizing flux due to the BAL absorption. If the latter is true, then the recombination timescale is larger than the 2655 days between observations, which could be used to estimate a density and distance provided photoionization modeling.

\section{Conclusions}

Our goal was to explore the Dunn et al. (2007) FUSE survey in depth for correlations and variability. We sought to characterize the intrinsic absorption seen in nearly $50 \%$ of all nearby active galaxies in order to help characterize the outflow from the nuclear engine.

As shown by Laor \& Brandt (2002), there is a relation between the maximum outflow velocity and the luminosity. Their survey contained moderate redshift objects and BAL quasars, while our survey was for smaller redshifts and luminosities. However, as shown previously, our relation between $\mathrm{v}_{\max }$ and $\mathrm{L}$ is consistent with theirs. In both our surveys there is a distinct upper limit on the maximum velocity dependent upon the luminosity of the object. This leads us to believe that both classical narrow intrinsic absorption lines and broad absorption lines are governed by the same relationship, thus they may be related, in the sense that they may share a common driving mechanism. Due to their simultaneous presence in WPVS 007 and PG 1411+442 however, we feel that the nature of their origins may be different.

Laor \& Brandt (2002) also found for higher redshift galaxies that EW \& luminosity are correlated for C IV. We examined correlations between the observed lines and the AGN properties. We found no link between the EW and the luminosity. Because EW increases with column density of the particular ion in question, it would seem that an increase in flux would increase the O VI EW in cases where the ionization parameter is low and vise versa for a highly ionized cloud, at least until the line reaches heavy saturation. We do not see this trend for either O VI or Ly $\beta$. This is most likely due to the heavy saturation in O VI and even Ly $\beta$ in many cases while $\mathrm{C}$ IV and $\mathrm{N} \mathrm{V}$ do not saturate as easily.

It has been seen in the past that some objects show bulk transverse motion along with the more easily detectable radial motion (Crenshaw et al. 2003). Of the 35 objects available we have 22 with more than one observation. Two of these objects have only one usable observation, leaving 20 total useful data sets. In the survey we find 8 that show change. The frequency of variability events seems to be low, in the FUSE data. With 22 available objects, 
only 8 show variability. This could mean the events are rare, that only weak absorption is subject to this change and/or the sample is biased by small number statistics, or the O VI saturation makes detecting variability difficult.

None of our objects with EW changes can be explained with transverse bulk motion. We have 7 that can be explained with ionization changes. In the case of Mrk 509 with modeling of the subcomponents, we can find a distance and density $\left(\mathrm{n}_{H}\right)$ of the third subcomponent of $0.3 \mathrm{pc}$ and $3.8 \times 10^{8} \mathrm{~cm}^{-1}$, respectively. Also, we find one object that shows evidence for radial velocity changes (PG 0804+761); we currently have no explanation for this.

We find in Mrk 817 that the absorption appears in relatively weak lines, and furthermore even the changes in the absorption features for Mrk 509 are in relatively weak lines. This is the case for most transversely moving clouds seen in the past. With the low signal-to-noise FUSE data it may be that these weak lines are swamped by the surrounding noise and only strong absorption features are noticeable, or it may be that bulk transverse motion is not a common event. Given that these changes are in weak absorbers, it suggests that strong absorbers appear more static in the far UV than weak absorbers due to saturation and blending in many of the lines.

This research has made use of the NASA/IPAC Extragalactic Database (NED) which is operated by the Jet Propulsion Laboratory, California Institute of Technology, under contract with the National Aeronautics and Space Administration. This research has also made use of NASA's Astrophysics Data System Abstract Service. We acknowledge support of this research under NASA grants NNG05GC55G, NNG06G185G and NAG5-13109. 


\section{REFERENCES}

Anderson, K. S., \& Kraft, R. P. 1969, ApJ, 158, 859

Arav, N., Li, Z.-Y. \& Begelman, M. C. 1994, ApJ, 432, 62

Arav, N., Korista, K. T. \& de Kool, M. 2002, ApJ, 566, 699

Begelman, M. C., McKee, C. F., \& Shields, G. A. 1983, ApJ, 271, 70

Blandford R. D. \& Payne D. G. 1982, MNRAS, 199, 883

Boroson T. A. \& Green R. F. 1992, ApJS, 80, 109

Botte, V., Ciroi, S., Rafanelli, P. \& Di Mille, F. 2004, AJ, 127, 3168

Crenshaw, D. M., Kraemer, S. B., Boggess, A., Maran, S. P., Mushotzky, R. F., \& Wu, C.-C. 1999, ApJ, 516, 750

Crenshaw, D. M., Kraemer, S.B., \& George, I.M. 2003, ARA\&A, 41, 117

Cromwell, R., \& Weymann, R. 1970, ApJ, 159, 147

Dixon, W. V. D., Kruk, J. \& Murphy, E. 2002, The CalFUSE Pipeline Reference Guide

deKool, M. \& Begelman, M. C. 1995, ApJ, 455, 448

Dunn, J. P., Jackson, B., Deo, R. P., Farrington, C., Das, V., \& Crenshaw, D. M. 2006, PASP, 118, 572

Dunn, J. P.,Crenshaw, D. M., Kraemer, S.B., \& Gabel, J. R. 2007, AJ, 134, 1061 (Paper I)

Feldman, P. D., Sahnow, D. J., Kruk, J. W., Murphy, E. M. \& Moos, H. W. 2001, JGR, 106.8119

Foltz, C. B., Chaffee, F. H., Hewett, P. C., Weymann, R. J., \& Morris, S. L. 1990, AAS, 22, 806

Gabel, J. et al. 2005, ApJ, 631, 741

Gabel, J. et al. 2003, ApJ, 595, 120

Ganguly, R., Masiero, J., Charlton, J. C., Sembach, K. R. 2003, ApJ, 598, 922

Ganguly, R., Brotherton, M. S., Cales, S., Scoggins, B., Shang, Z., \& Vestergaard, M. 2007, 665, 990

Ganguly, R. \& Brotherton, M. S. 2008, ApJ, 672, 102

Gillmon, K., Shull, J. M., Tumlinson, J. \& Danforth, C. 2006, ApJ, 636, 891

Grupe, D. \& Mathur, S. 2004, ApJ, 606, 41

Khachikian \& Weedman 1974, ApJ, 192, 581 
Kaspi et al. 2000, ApJ, 533, 631

Kraemer, S. B., Turner, T. J., Crenshaw, D. M. \& George, I. M. 1999, ApJ, 519, 69

Kraemer, S. B., Crenshaw, D. M., \& Gabel, J. R. 2001, ApJ, 557, 30

Kraemer, S. B., Crenshaw, D. M., Yaqoob, T., McKernan, B., Gabel, J. R., George, I. M., Turner, T. J. \& Dunn, J. P. 2002, ApJ, 582, 125

Kraemer et al. 2003, ApJ, 582, 125

Kraemer et al. 2005, ApJ, 633, 693

Kraemer, S. B., Crenshaw, D. M., Gabel, J. R., Kriss, G. A., Netzer, H., Peterson, B. M., George, I. M., Gull, T. R., Hutchings, J. B., Mushotzky, R. F. \& Turner, T. J. 2006, ApJS, 167, 161

Kriss, G. A. 2002, ASPC, 255, 69

Krolik, J. H. \& Kriss, G. A. 1995 ApJ, 447,512

Laor, A. \& Brandt, W. N. 2002, ApJ, 569, 641

Mason et al. 1995, MNRAS, 274, 1194

Maran, S. P., Crenshaw, D. M., Mushotzky, R. F., Reichert, G. A., Carpenter, K. G., Smith, A. M., Hutchings, J. B., Weymann, R. J. 1996, ApJ, 465, 733

Marziani, P., Sulentic, J. W., Zamanov, R., Calvani, M., Dultzin-Hacyan, D., Bachev, R. \& Zwitter, T. 2003 ApjS, 145, 199

McLure, R. J. \& Dunlop, J. S. 2004, MNRAS, 352, 1390

Morton, D. C. 1991, ApJS, 77, 119

Osterbrock, D. E. 1989, Astrophysics of gaseous nebulae and active galactic nuclei (Research supported by the University of California, John Simon Guggenheim Memorial Foundation, University of Minnesota, et al. Mill Valley, CA, University Science Books, 1989, 422 p.)

Penton, S. V., Stocke, J. T. \& Shull, J. M. 2002 ApJ, 565, 720

Peterson, B. M., Wanders, I., Bertram, R., Hunley, J. F., Pogge, R. W., \& Wagner, R. M. 1998, ApJ, 501, 82

Peterson et al. 2004, ApJ, 613, 682

Sahnow et al. 2000, ApJ, 538, 7

Sahnow, D. 2002, The FUSE Instrument and Data Handbook

Turnshek, D. A., Grillmair, C. J., Foltz, C. B. \& Weymann, R. J. 1988, ApJ, 325, 651 
Vestergaard, M. \& Peterson B. M. 2006, ApJ, 641, 689

Weymann, R. J., Carswell, R. F., \& Smith, M. G. 1981, ARA \& A, 19, 41

Weymann, R. J., Morris, S. L., Gray, M. E., Hutchings, J. B. 1997,ApJ, 483, 717

Xu, D. W., Komossa, Stefanie, Wei, J. Y., Qian, Y. \& Zheng, X. Z. 2003, ApJ, 590, 73 
Fig. 1. - New spectra taken at CTIO, plotted in the observed frame.

Fig. 2.- The relation between luminosity and velocity using only data from FUSE.

Fig. 3.- Extended plot of luminosity and velocity using data measured by Laor \& Brandt (2002). Crosses are data from our FUSE observations and asterisks are from the Laor \& Brandt (2002) survey.

Fig. 4.- Relationship between Eddington Ratio and maximum outflow velocity. Crosses are objects with reverberation mapping.

Fig. 5.- EW vs. specific luminosity for our sample. The top plot is for O VI $\lambda 1032$, the middle is for O VI $\lambda 1037$ and the bottom plot is for Ly $\beta$. Plus symbols are components labeled 1 from Paper I and asterisks any component other than the first.

Fig. 6.- FWHM vs.specific luminosity. The top plot is for O VI $\lambda 1032$, the middle is for O VI $\lambda 1037$ and the bottom plot is for Ly $\beta$.

Fig. 7. - The available spectra for Mrk 79 plotted in order of increasing time from bottom to top. The upper spectra are offset by: $6.0 \times 10^{-14} \& 1.2 \times 10^{-13} \mathrm{ergs} \mathrm{s}^{-1} \mathrm{~cm}^{-2} \AA^{-1}$. Component 1 (as identified by Paper I) is labeled by a dashed line and component 2 by a dotted and dashed line in each of the three lines $(\mathrm{Ly} \beta, \mathrm{O}$ VIb and O VIr in order of increasing wavelength).

Fig. 8. - This plot shows the relation for each of the three lines between EW, average velocity \& flux over time. Top to bottom the lines are plotted in order of decreasing wavelength. The dashed line represents the average value line of the data, which is mostly to guide the eye. The continuum seems to increase slightly while Ly $\beta$ and O VI 1038 seem to vary.

Fig. 9.- Plotted in chronological order from bottom to top. The earlier spectra for NGC 4151 show the flattening of the region between 1029 and 1032 Aby an additional absorption component. The offsets for these data are: $4.0 \times 10^{-13}, 8.0 \times 10^{-13} \& 1.2 \times 10^{-12} \mathrm{ergs} \mathrm{s}^{-1} \mathrm{~cm}^{-2}$ $\AA^{-1}$.

Fig. 10. - These spectra show the changes seen in Mrk 817. We see variability in the O VIb line, and find absorption appearing between 1051 and $1052 \AA$ and 1056 and 1057 $\AA$ (components 2 and 3 ). There is one point we were able to measure the continuum for, but the lines were lost in the $\mathrm{S} / \mathrm{N}$. We have included the lightcurve point for completeness.

Fig. 11.- Mrk 817 relationships of EW, velocity and flux for component 1over time. Both O VI lines show indications of decreasing EW with decreasing flux. 
Fig. 12.- We present the spectral time series for Mrk 279 with offsets in flux of: 1.0, 2.5, $3.5,5.0 \& 6.0 \times 10^{-13} \mathrm{ergs} \mathrm{s}^{-1} \mathrm{~cm}^{-2} \AA^{-1}$. This shows the variability seen in the lines.

Fig. 13.- Flux, EW and velocity against time for Mrk 279, similar to Figure 8. These quantities exhibit definite variability with time, and appears to be slightly anticorrelated with the flux. The last three points show signs of decrease in all three lines with the drop and rise of flux for those three observations, which hints at a lag in response time.

Fig. 14. - These are spectra of Mrk 290, which show the line trends with time (bottom to top). The offsets for these spectra are: $3.0 \times 10^{-14} \& 9.0 \times 10^{-14} \mathrm{ergs} \mathrm{s}^{-1} \mathrm{~cm}^{-2} \AA^{-1}$.

Fig. 15. - We see in these plots (similar to Figure 8), that there is an decrease of EW with an increase in flux for all three lines. The trend for Lyman $\beta$ is less convincing, however, due to the larger error bars.

Fig. 16. - We show the spectra for Mrk 509 and overplot the components 3 and 6 found by Kraemer et al. (2005), along with the dashed-dotted and dashed lines indicating our measured components 2 and 1 respectively. We have placed dotted lines to show the change in the slope of component 3 with respect to component 2 . The offsets for these are: $2.5 \&$ $5.0 \times 10^{-13} \operatorname{ergs~s}^{-1} \mathrm{~cm}^{-2} \AA^{-1}$.

Fig. 17.- Mrk 509 relations for component 1, plotted similar to Figure 8.

Fig. 18. - Mrk 509 relations for component 2, plotted similar to Figure 8.

Fig. 19.- Spectra for PG 0804+761. The lines are marked with the dashed average velocity line, and the lines clearly appear to move. The dotted line labels a galactic Fe II line $\lambda 1044.2$ to show that the centroid for the ISM lines are not moving. Offsets for these are: 2.5, 5.0, $7.5 \times 10^{-13} \operatorname{ergs~s}^{-1} \mathrm{~cm}^{-2} \AA^{-1}$.

Fig. 20.- PG 0804+761 relationships similar to Figure 8. We see definite correlations between velocity and EW with the flux levels with the exception of the Ly $\beta$ EW which seems to be low in the second observation.

Fig. 21. - Spectra showing the time evolution of NGC 3783. The measured component is labeled with the dashed line. Offsets for these are: $1.5,3.0,4.5,6.0,7.5 \& 9.0 \times 10^{-13}$ ergs $\mathrm{s}^{-1} \mathrm{~cm}^{-2} \AA^{-1}$.

Fig. 22.- This shows the relation for NGC 3783 between EW, velocity and flux over time. Similar to Figure 8. We see definite variability in the EW over time for all three lines. There appears to be a possible correlation between the change in $\mathrm{Ly} \beta \mathrm{EW}$ and continuum flux. 
Fig. 23.- Spectrum for WPVS 007. The three absorption features are marked with dashed lines. 


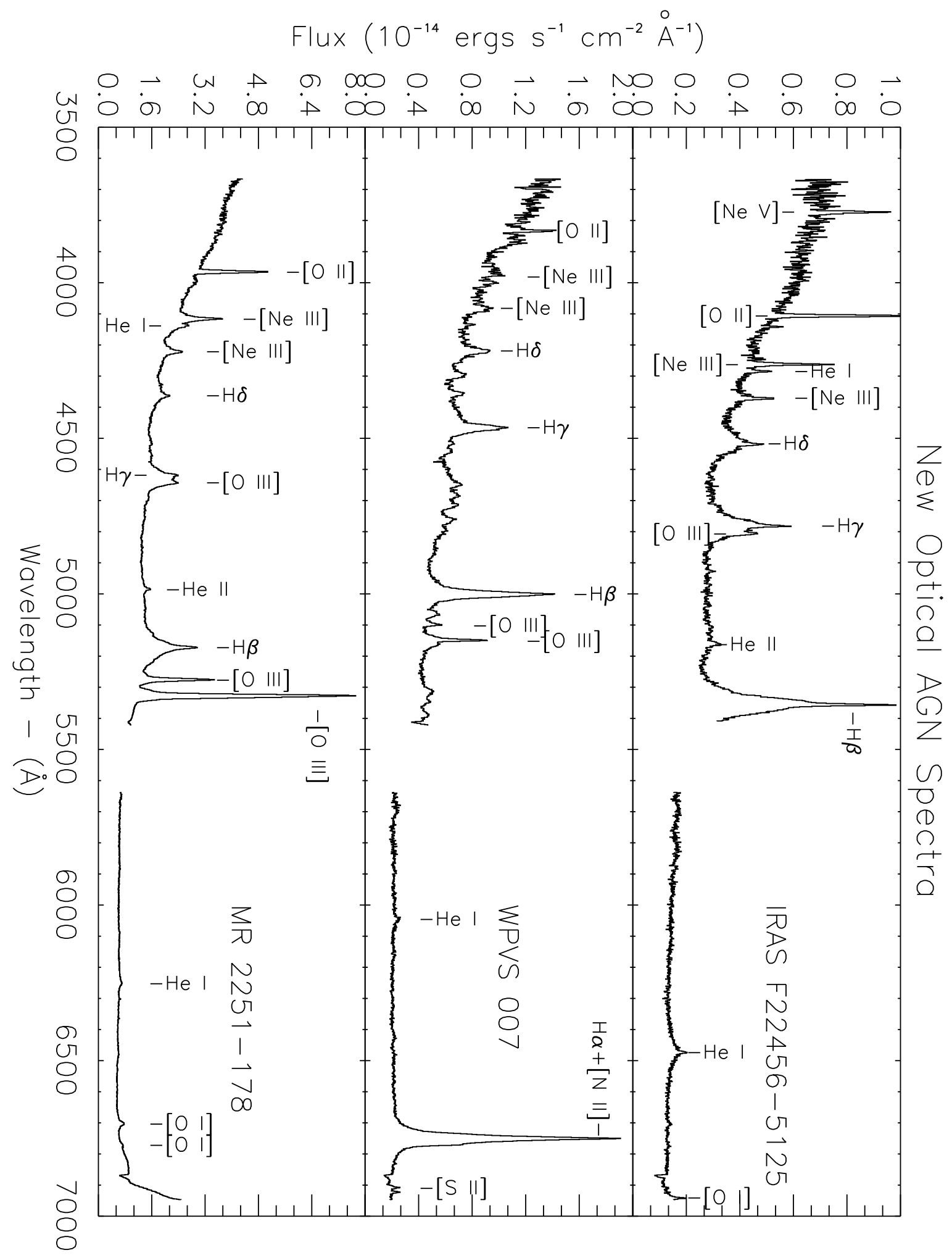

Fig. 1. 


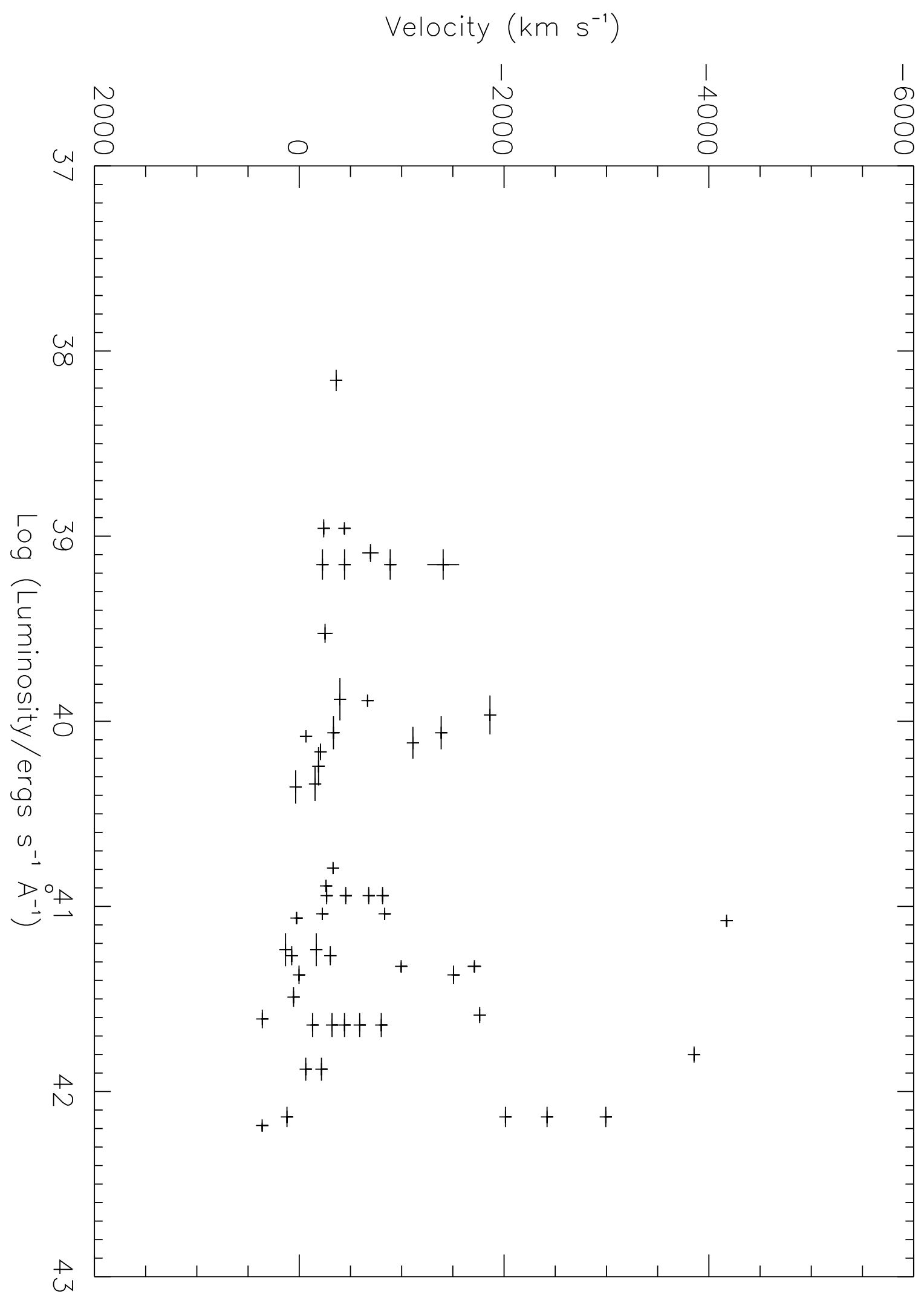

Fig. 2. 


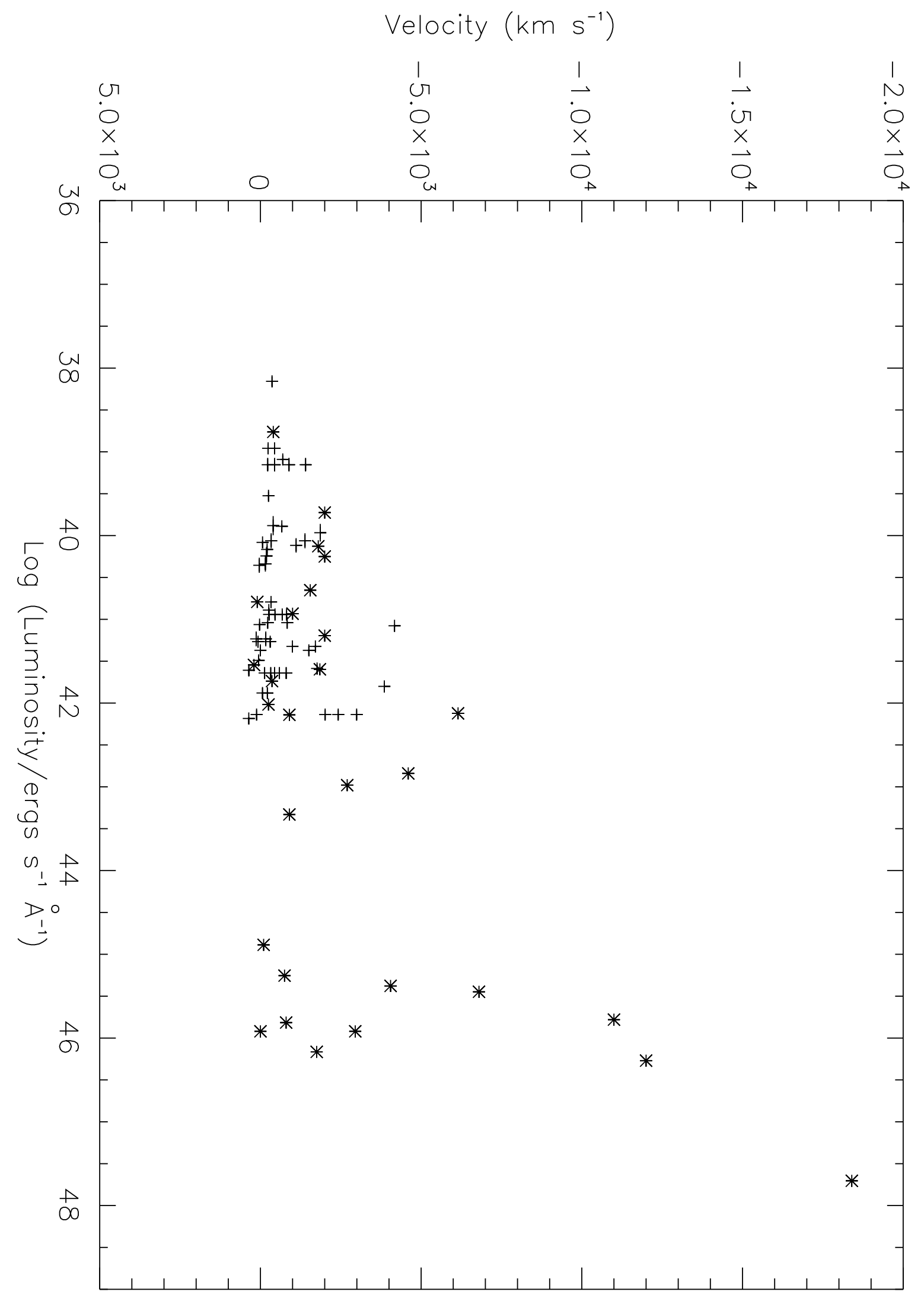

Fig. 3. 


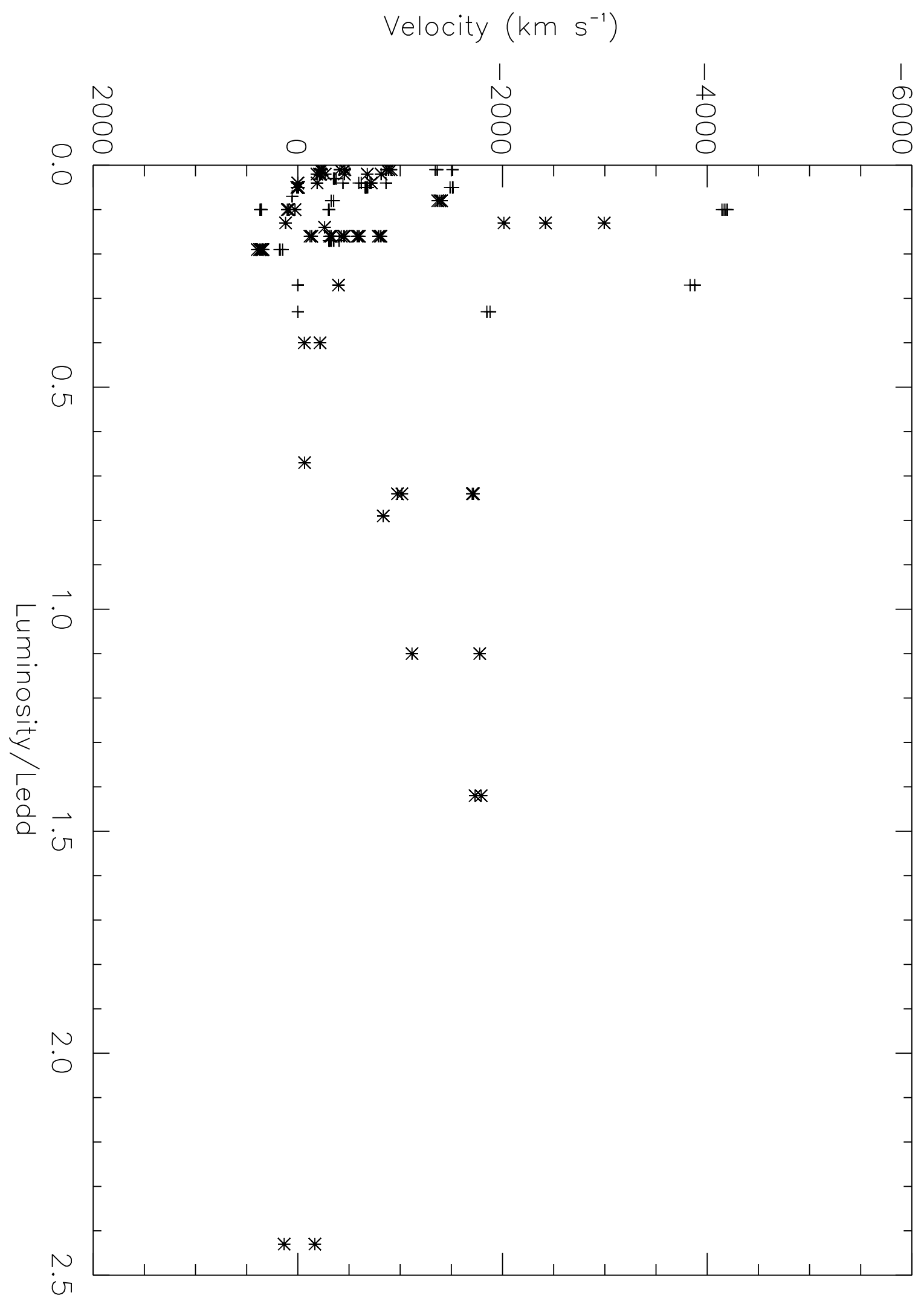

Fig. 4. 


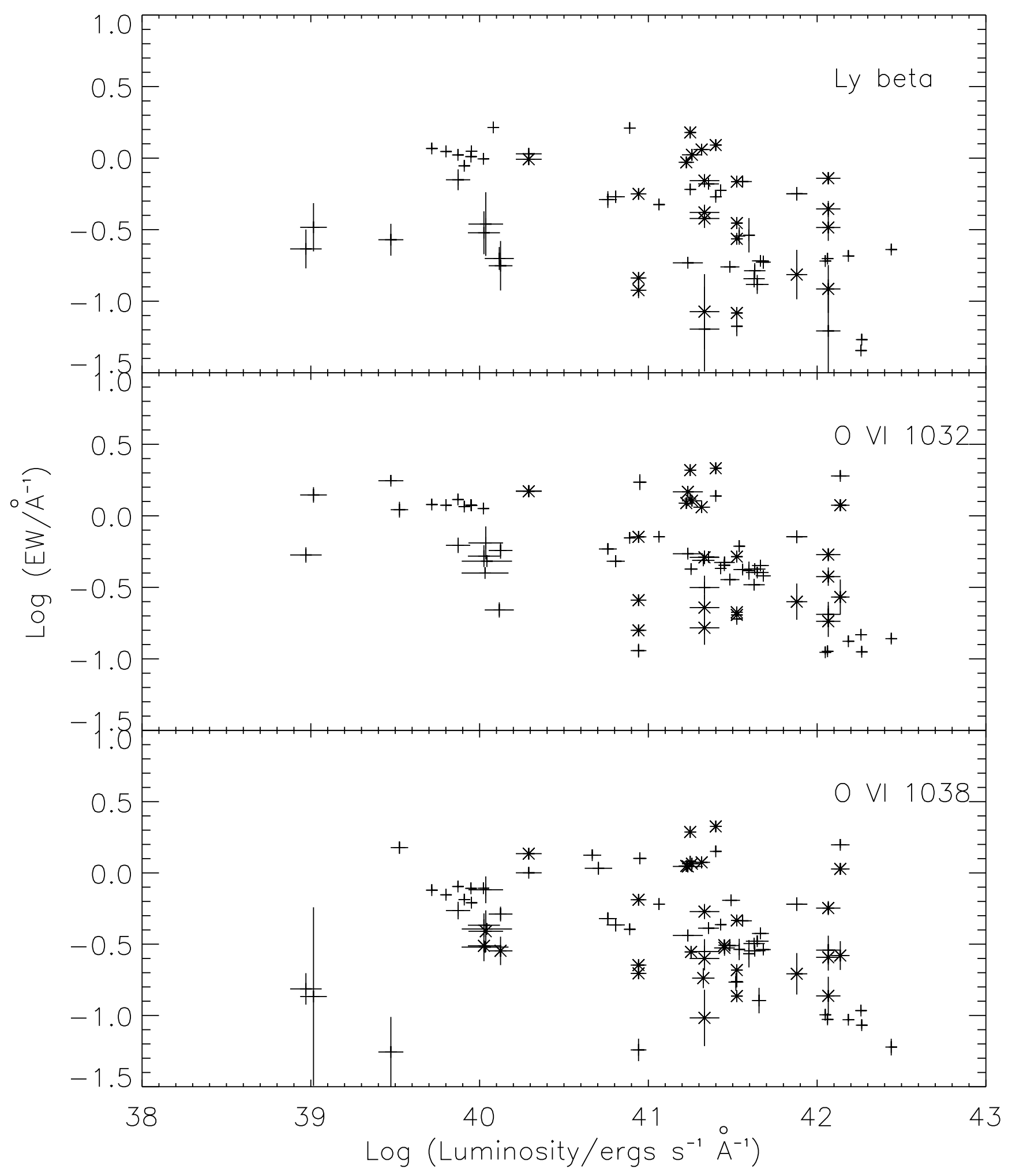

Fig. 5. 


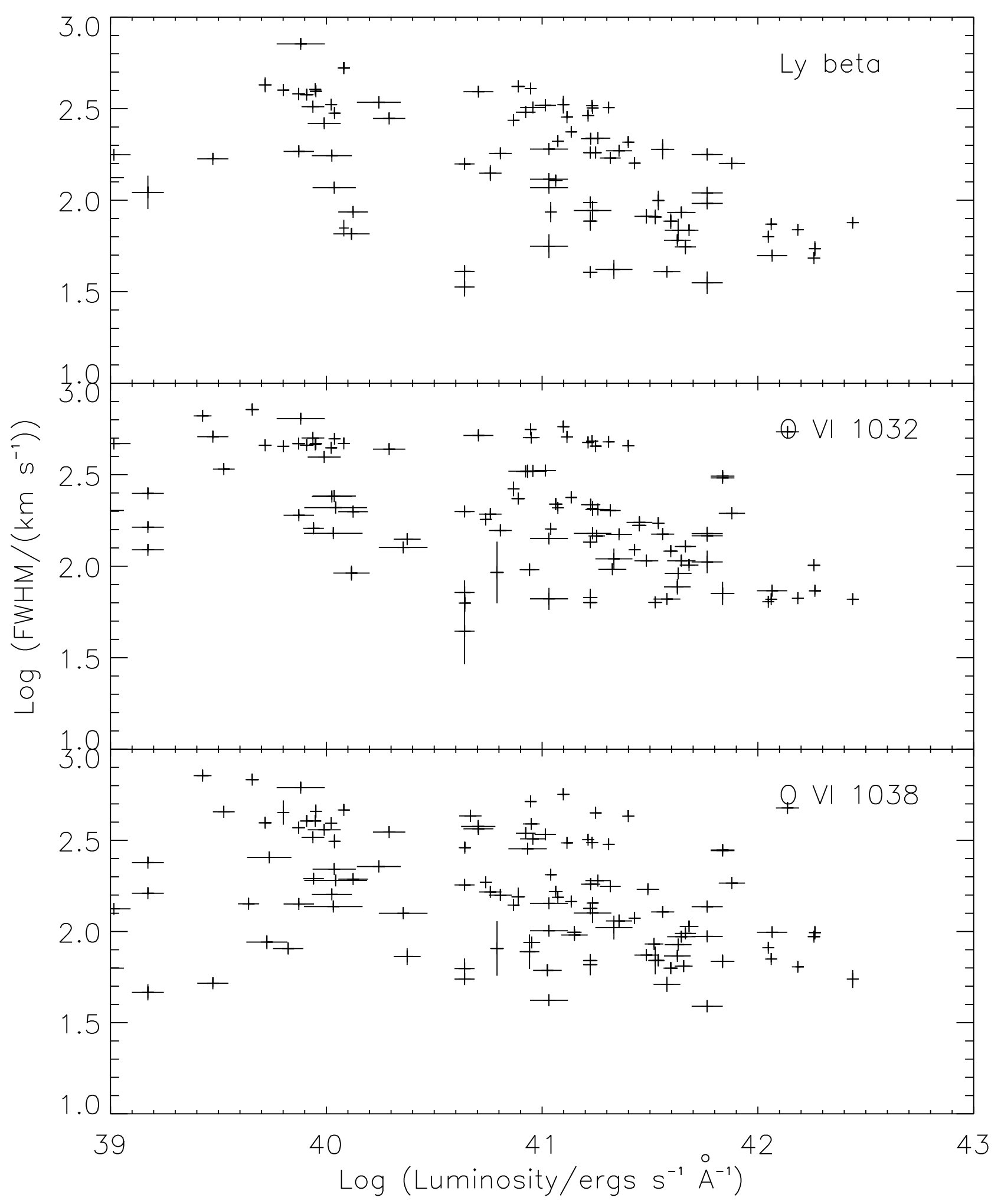

Fig, 6a. 


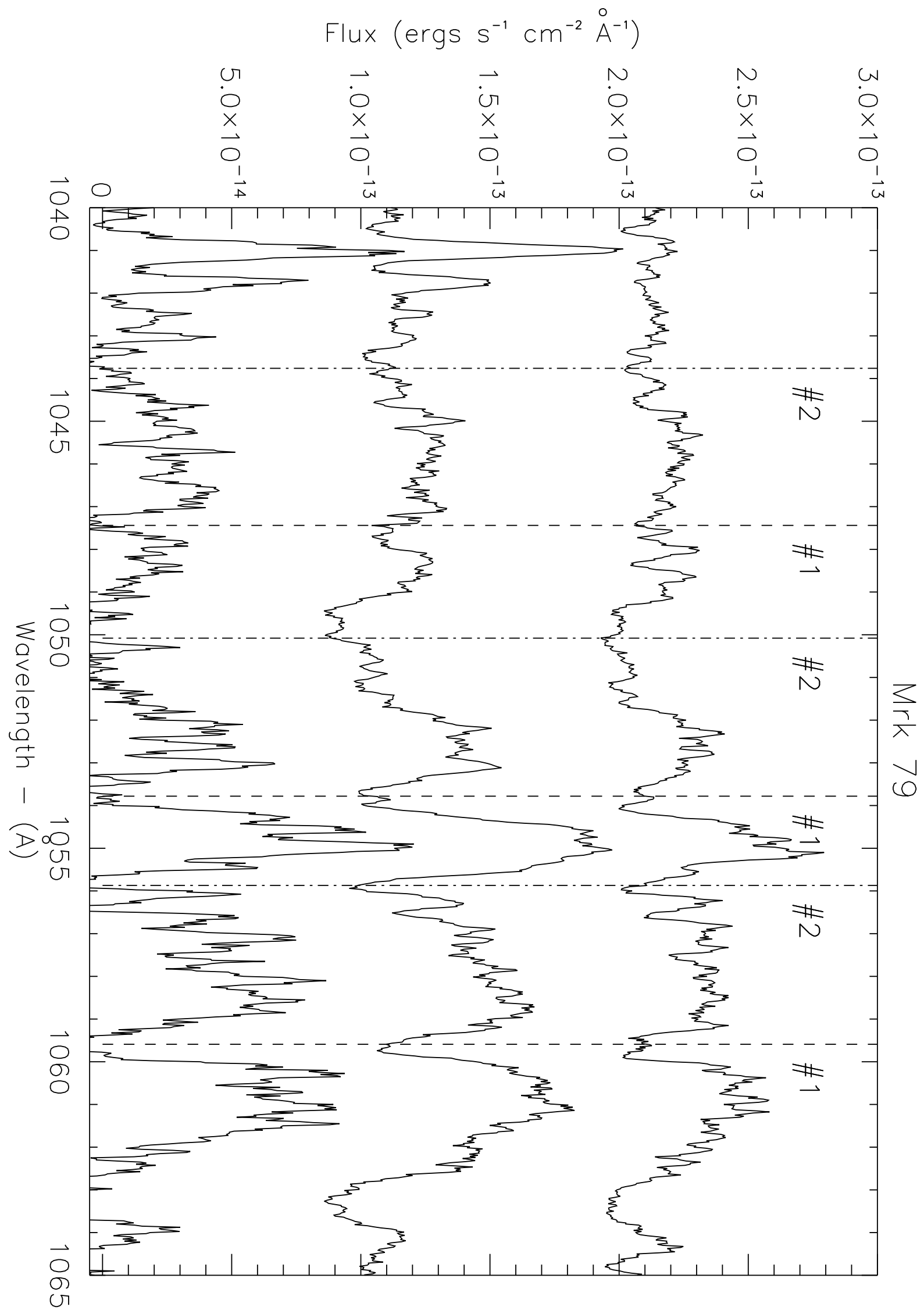

Fig. 7. 


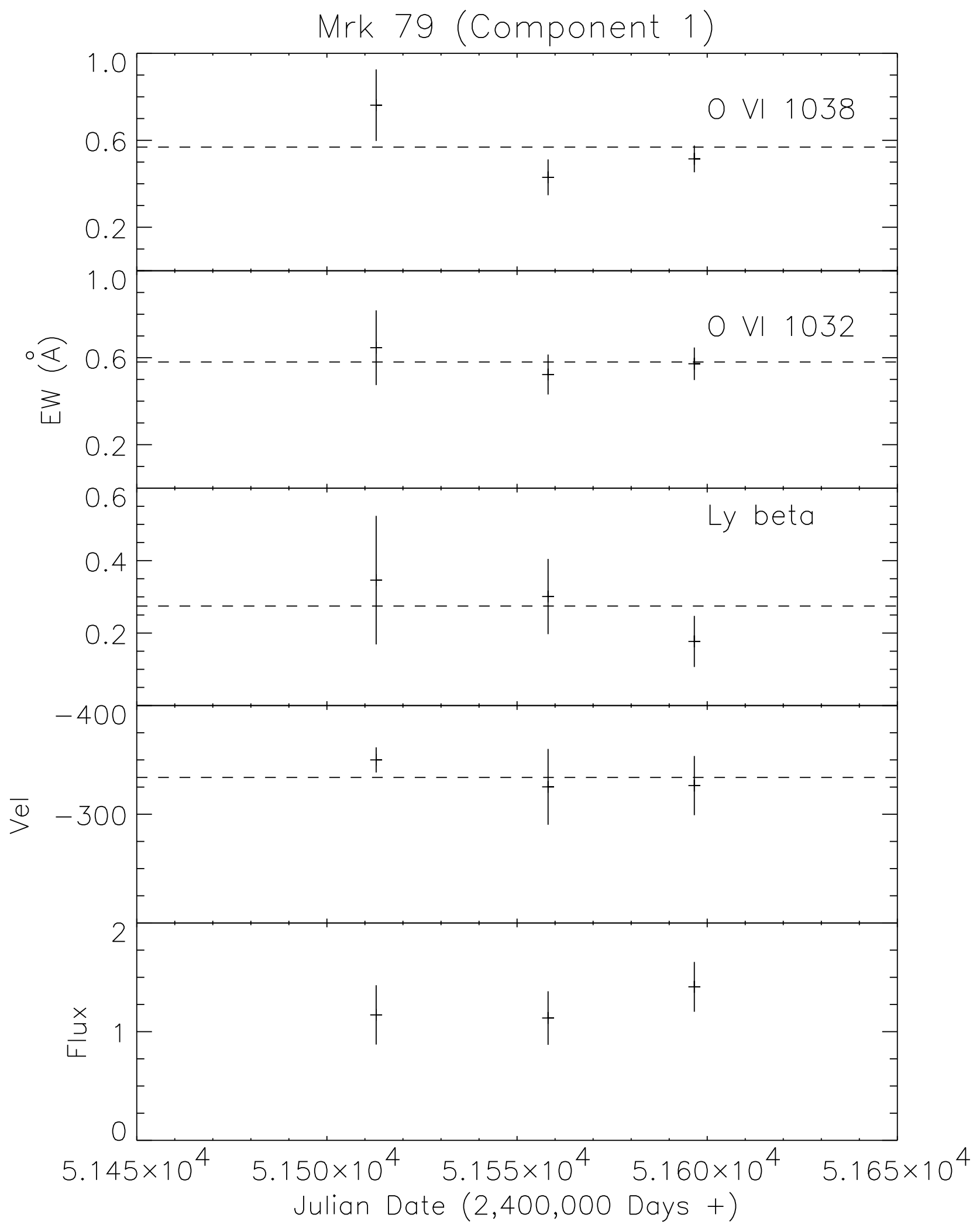

Fig. 8. 


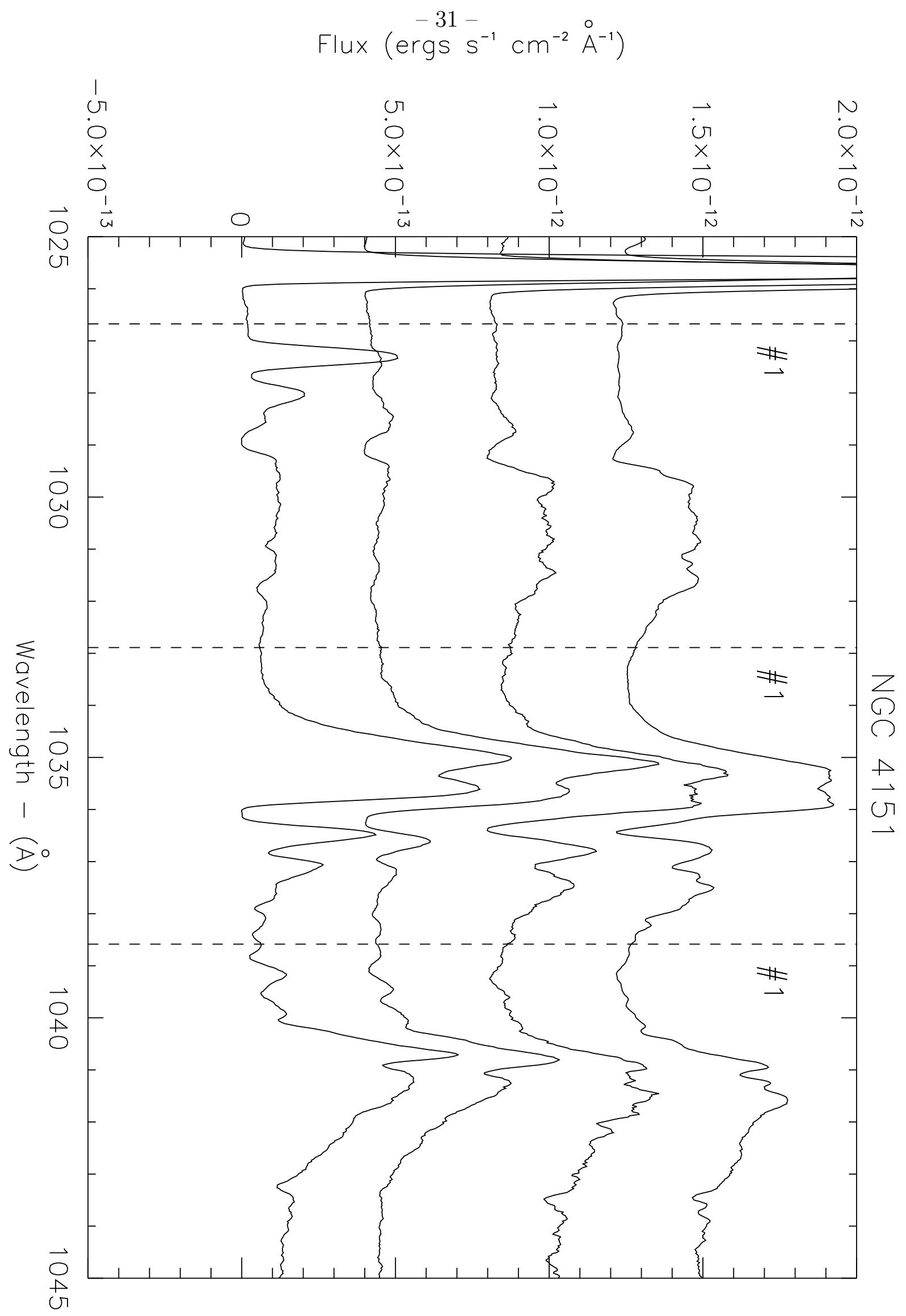

Fig. 9. 


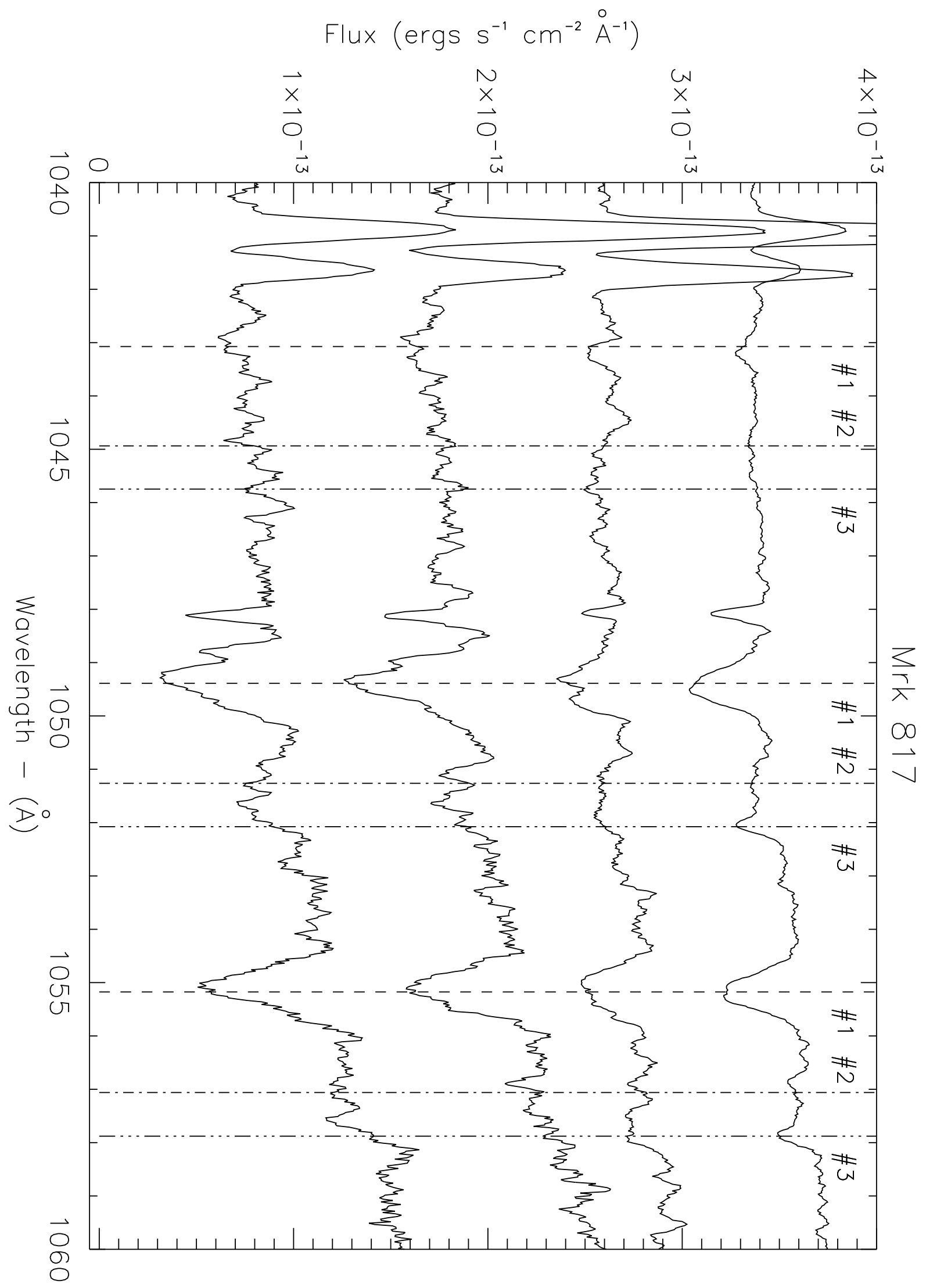

Fig. 10. 


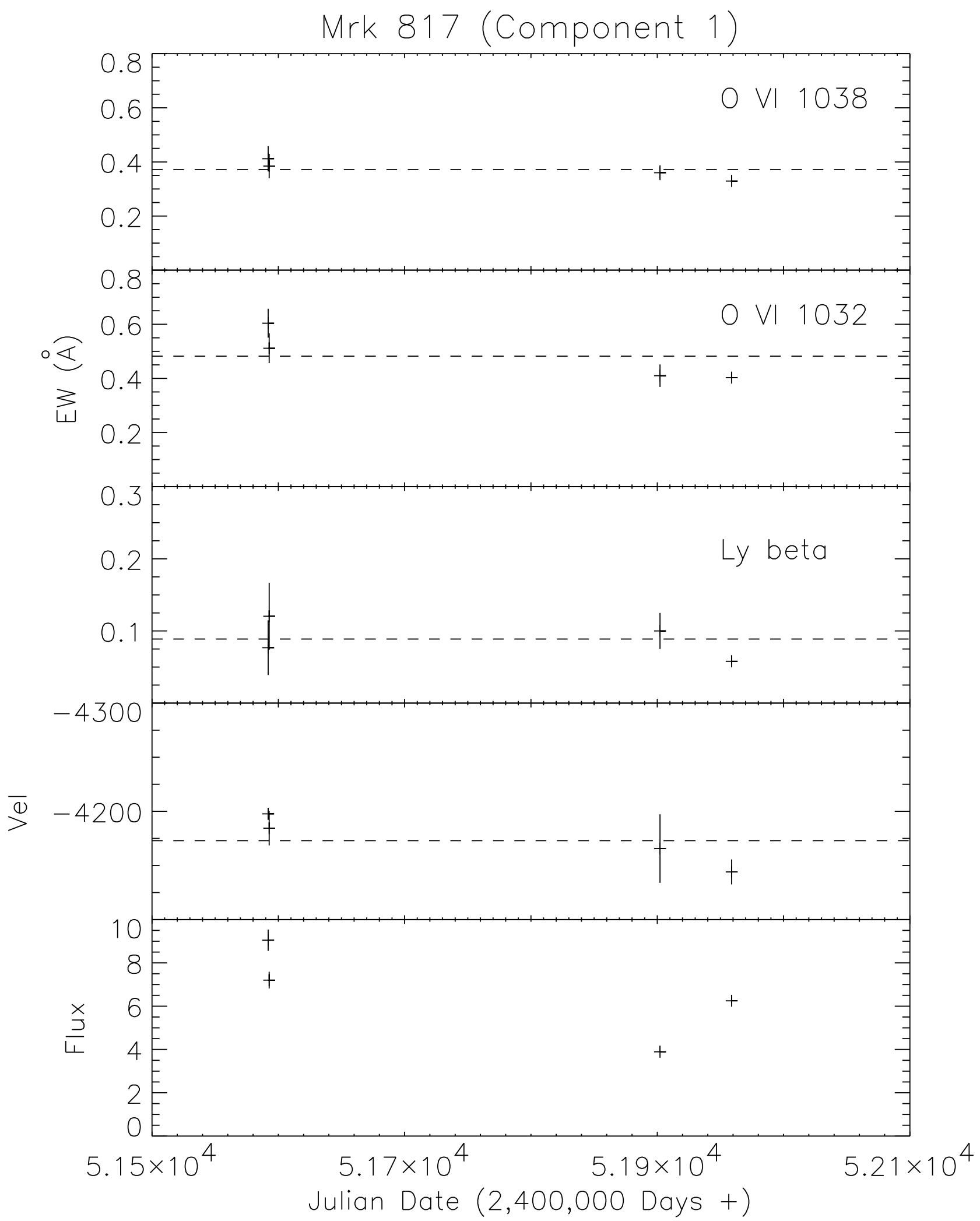

Fio. 11 


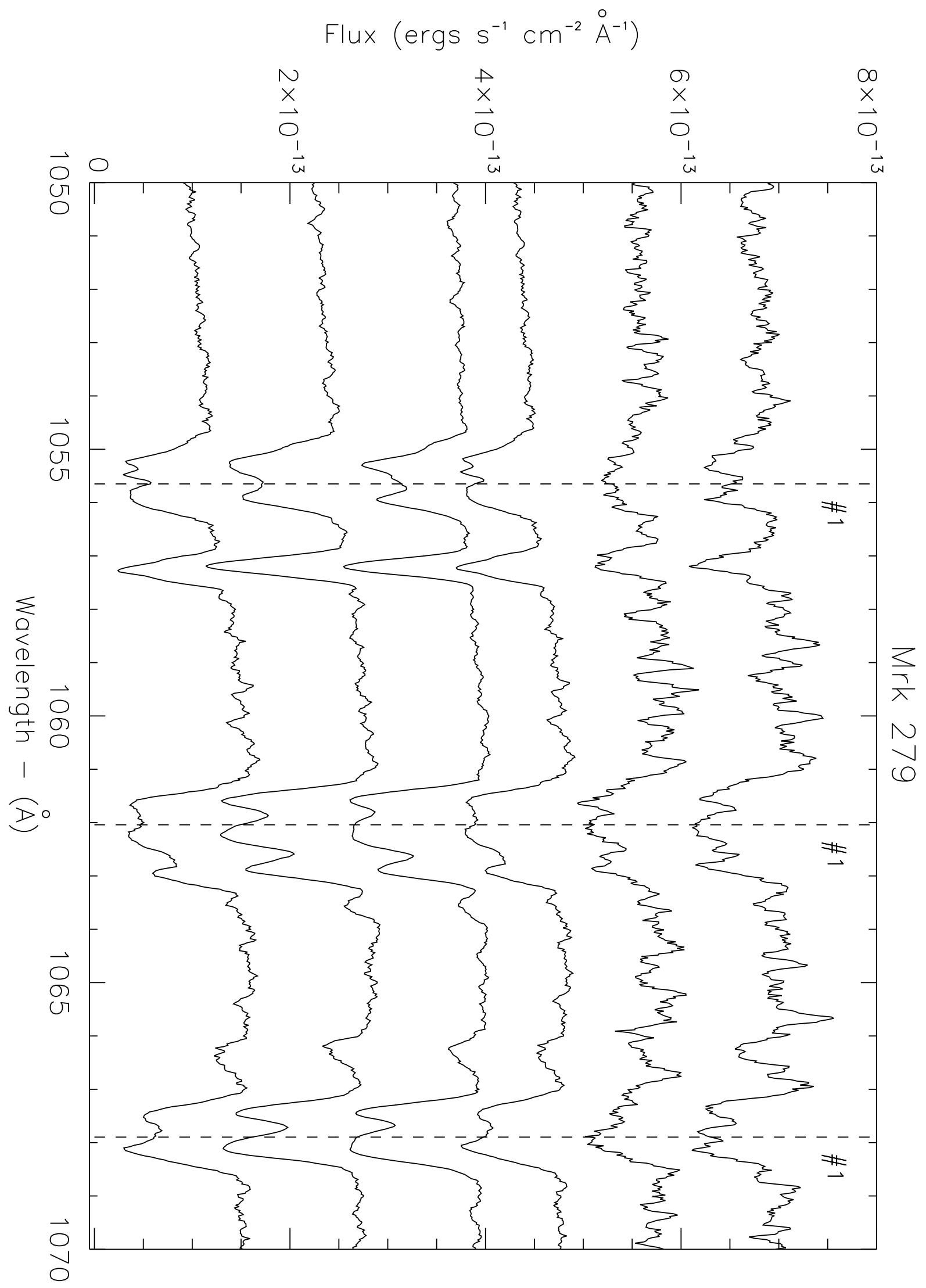

Fig. 12 . 


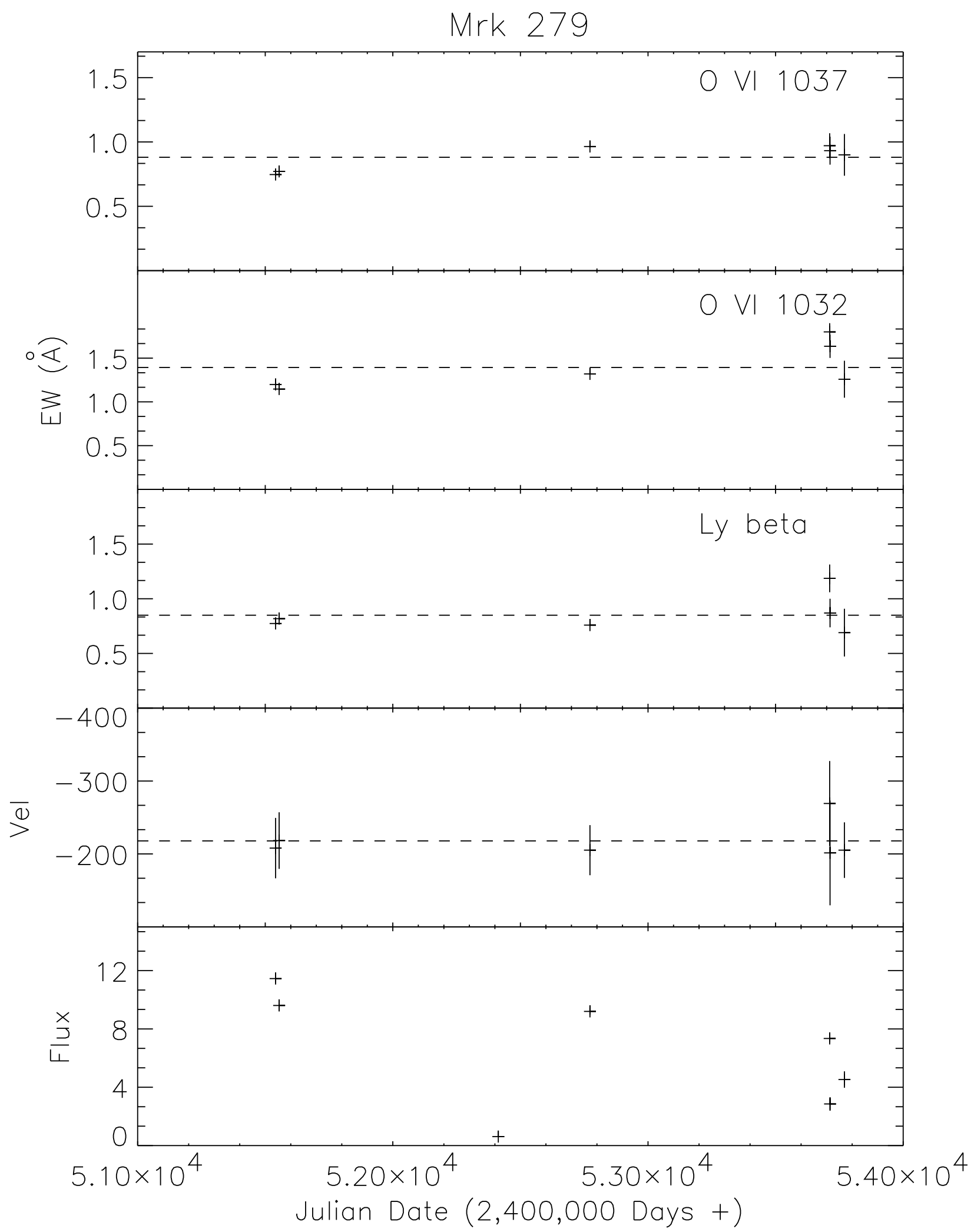

Fiq. 13. 


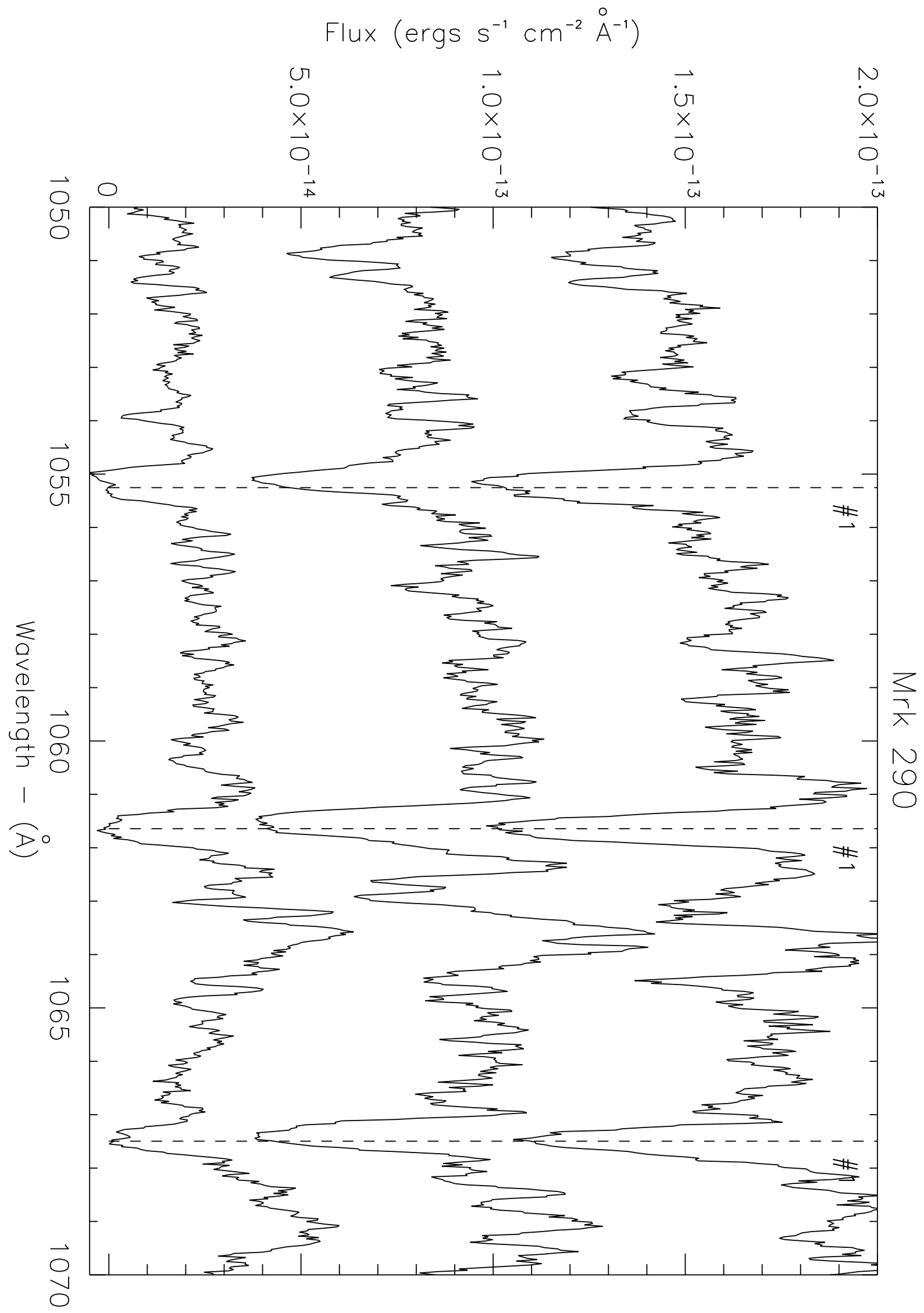

Fig. 14. 


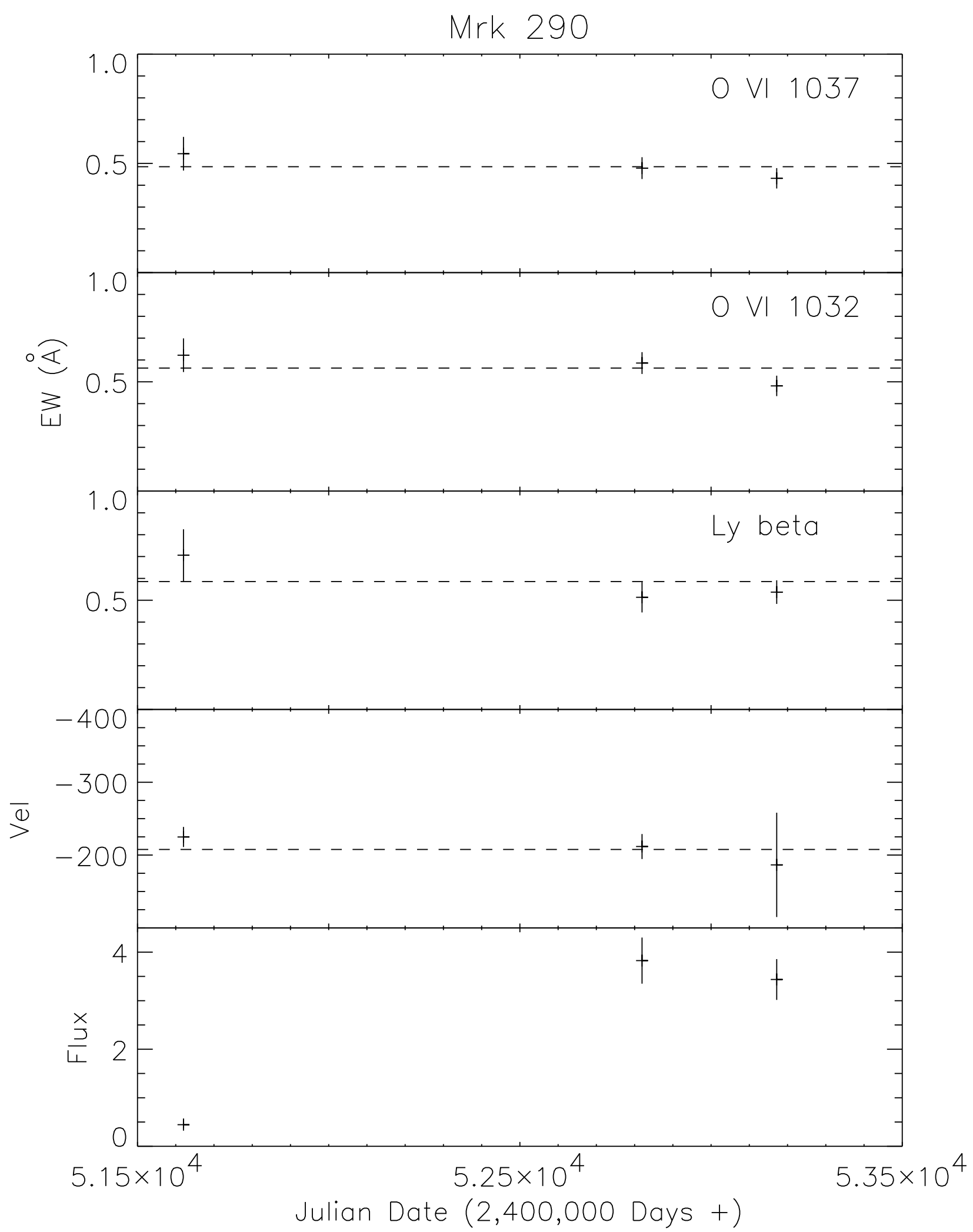

Fig. 15. 


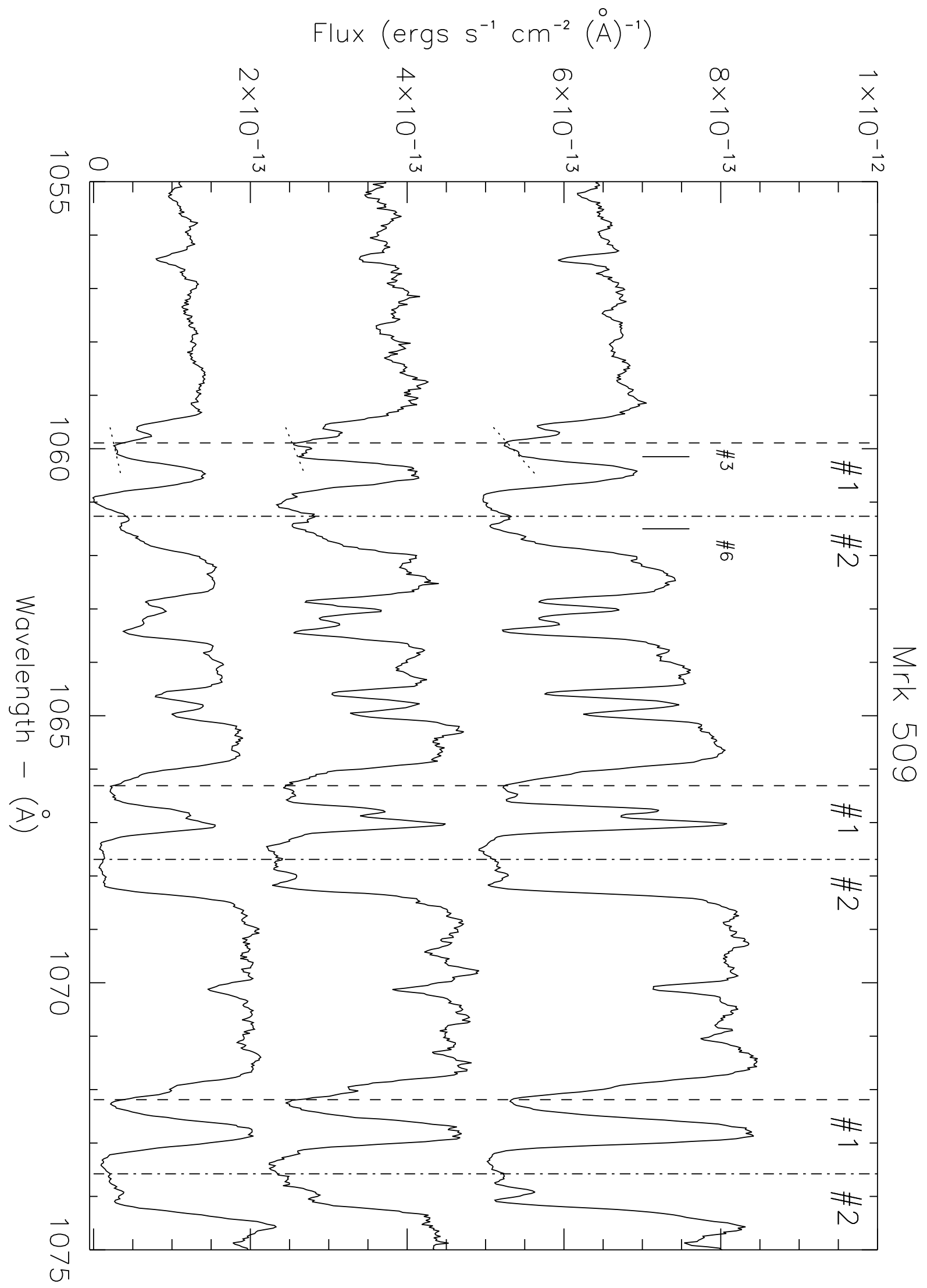

Fig. 16. 


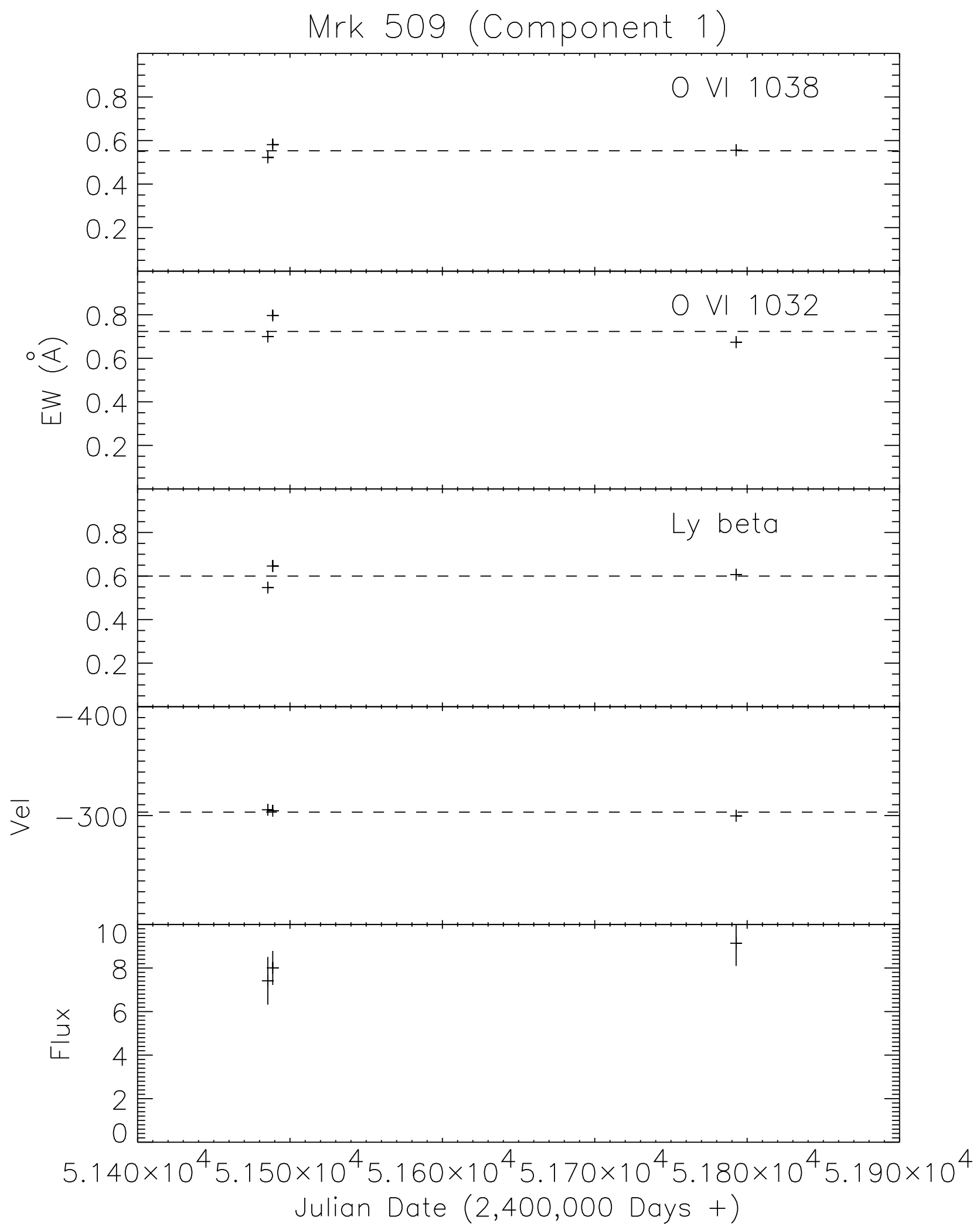

Fio. 17 


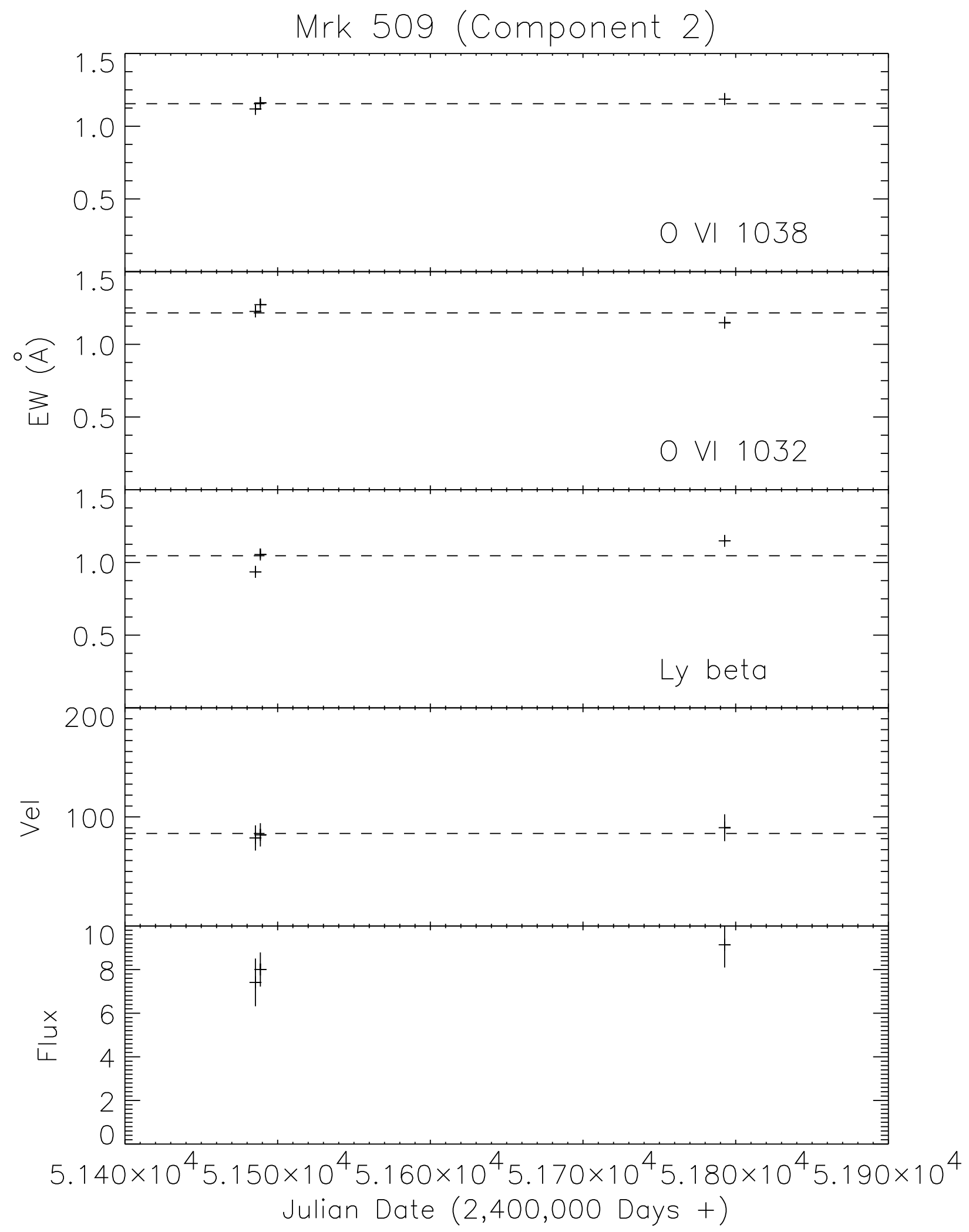

Fiq. 18 


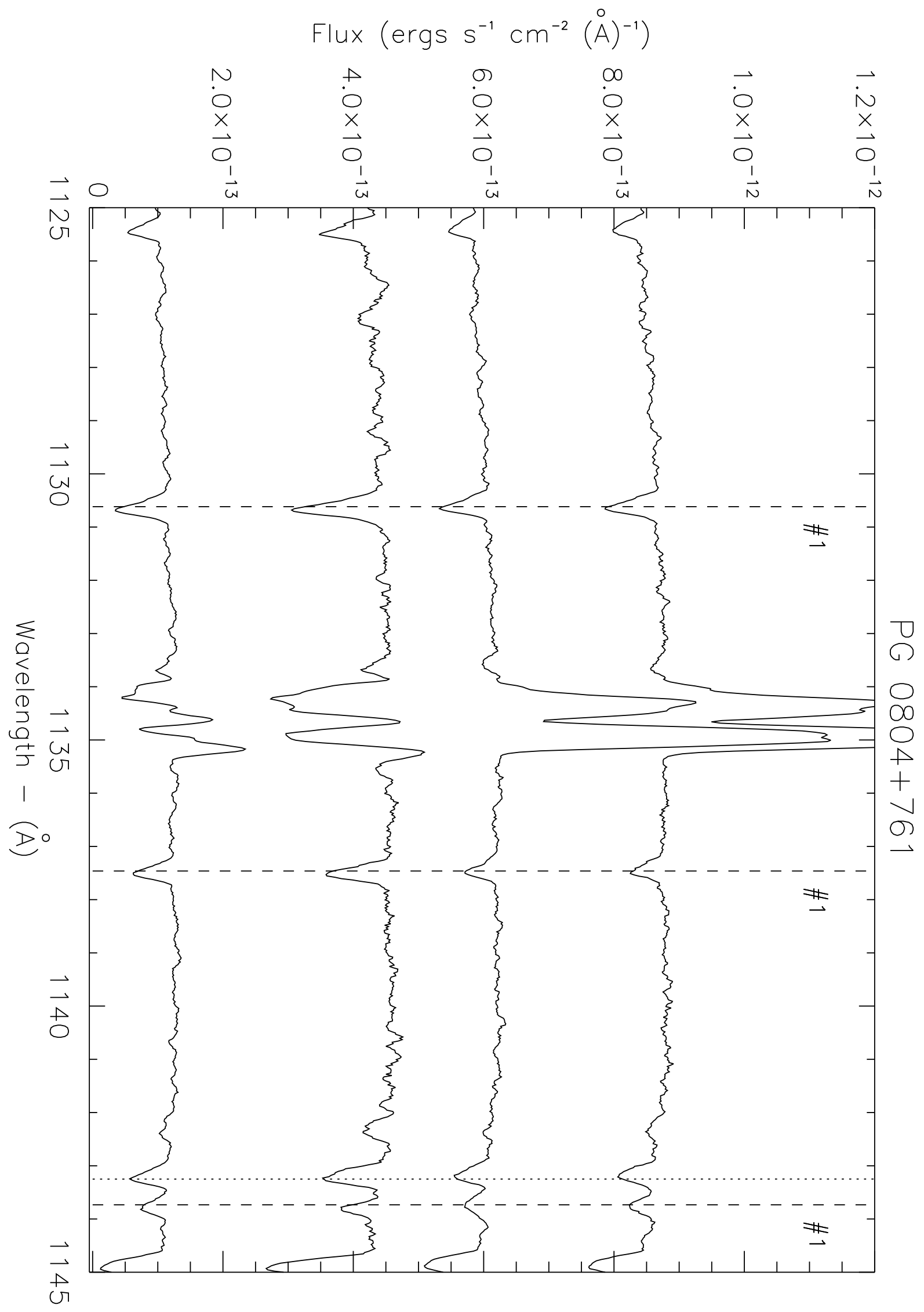

Fig. 19. 


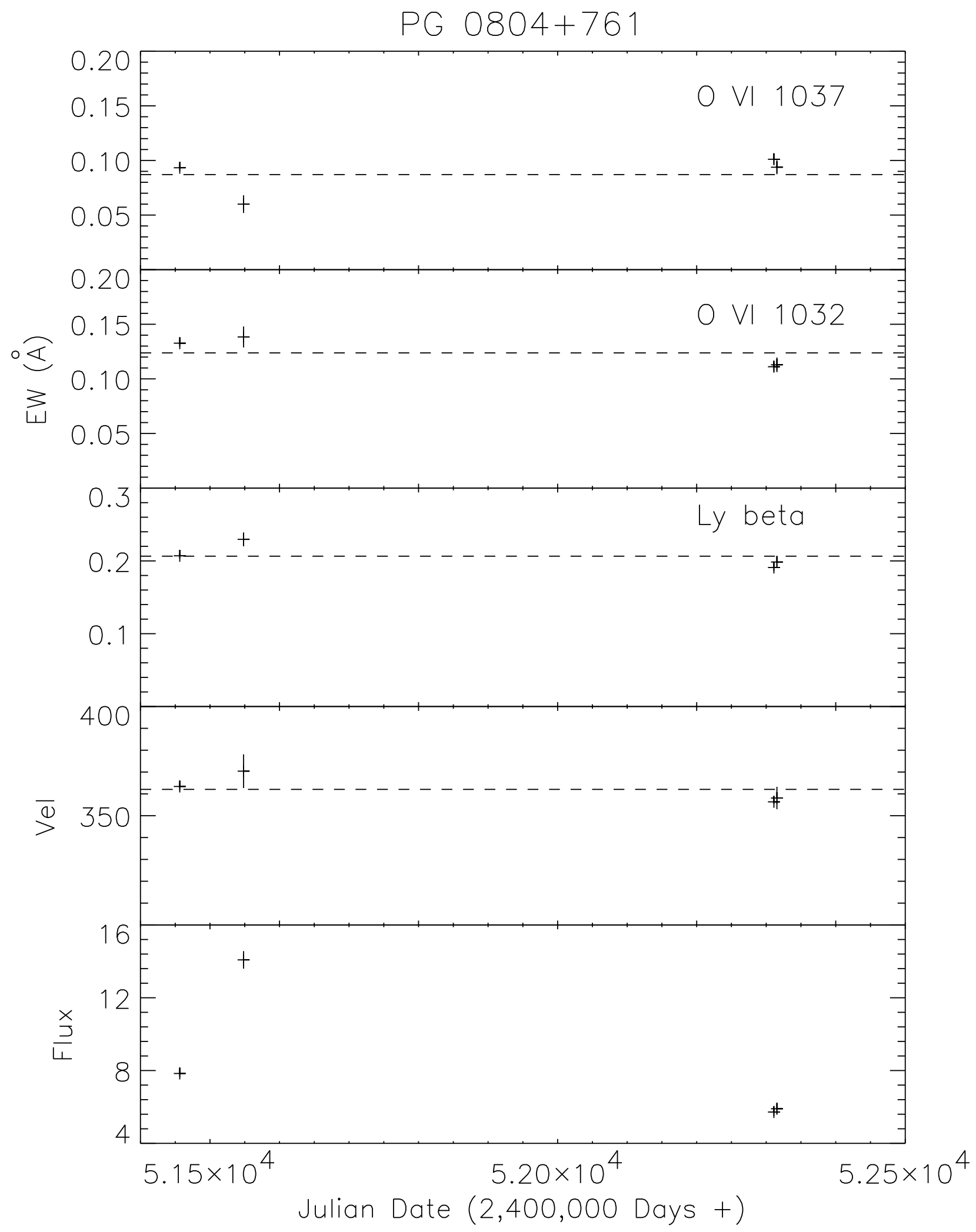

Fic, 20 


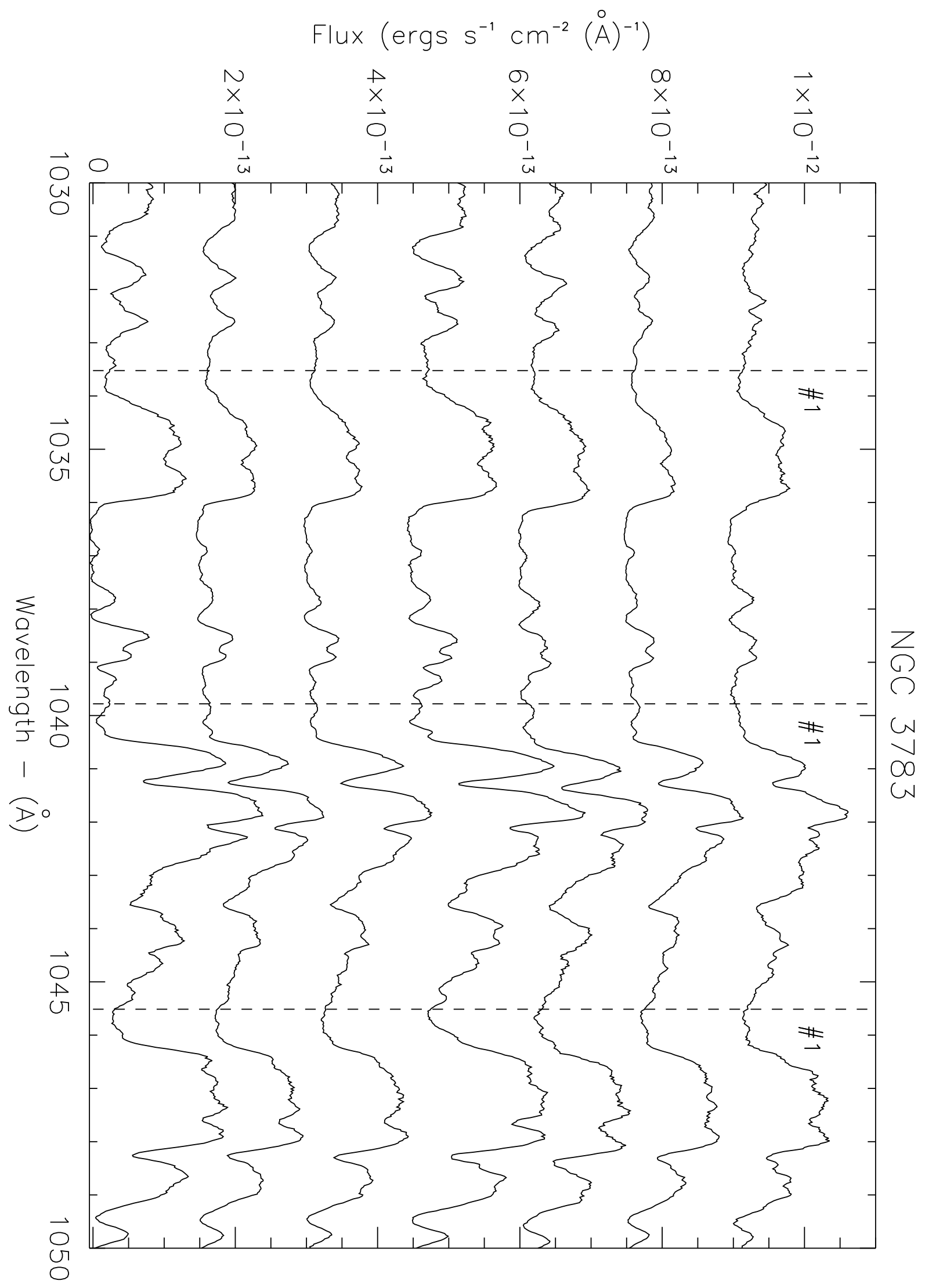

Fig. 21. 


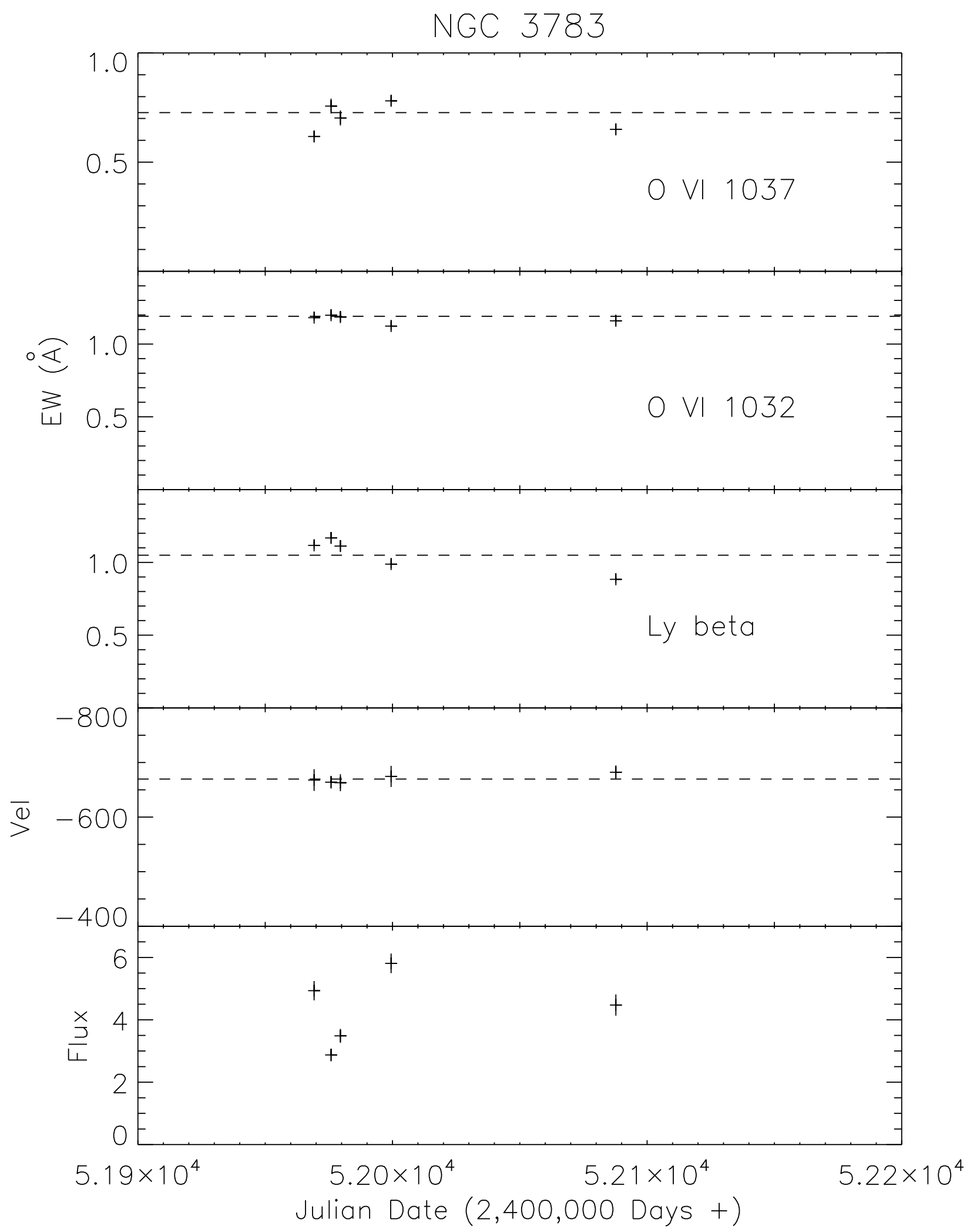

Fig. 22 


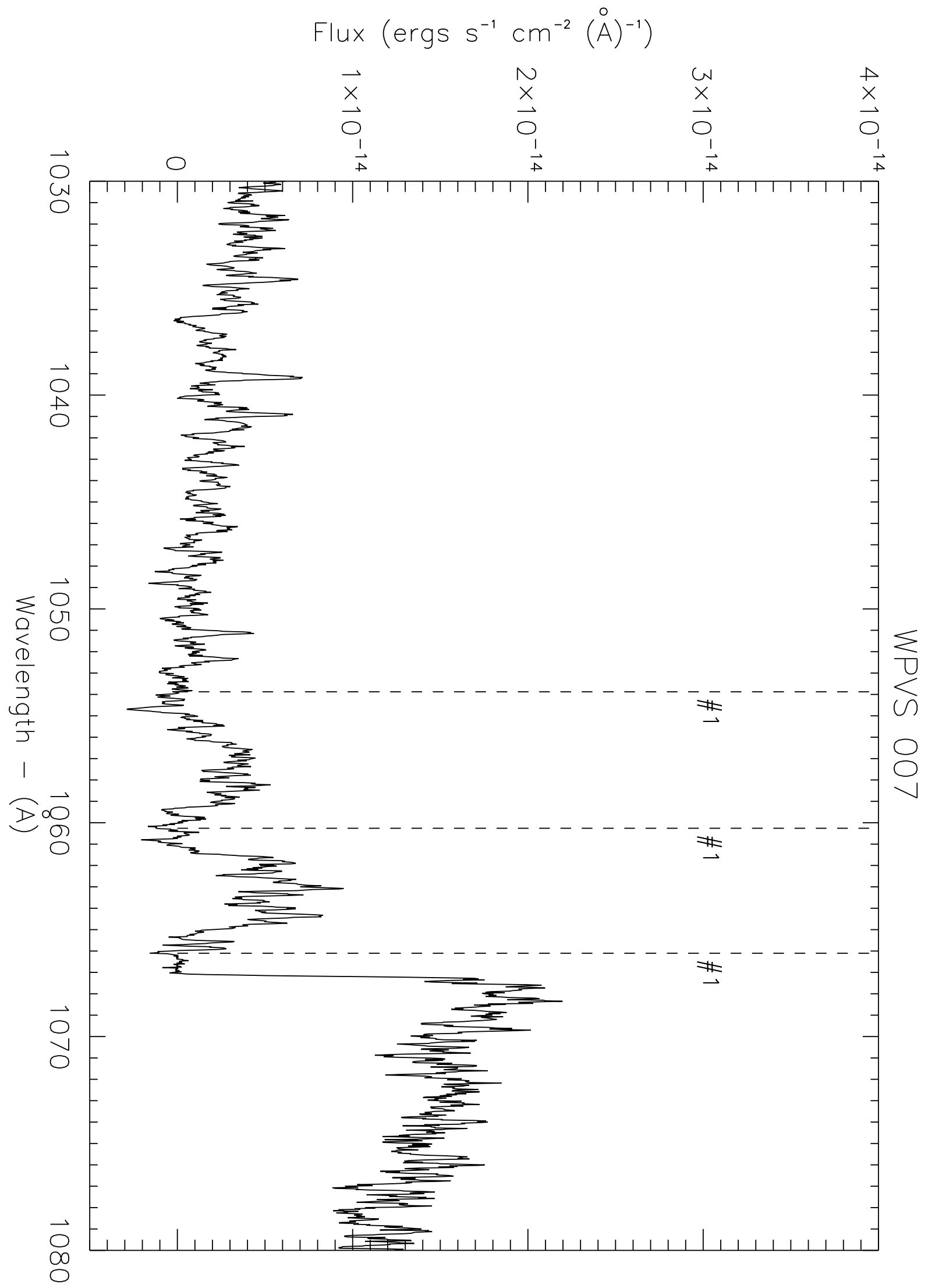

Fig. 23. 
Table 1. Observation Log for CTIO Spectra

\begin{tabular}{lcccc}
\hline \hline \multicolumn{1}{c}{ Object } & Date & $\begin{array}{c}\text { Integration Time } \\
(\mathrm{s})\end{array}$ & $\begin{array}{c}\text { Resolution FWHM } \\
(\AA)\end{array}$ & $\begin{array}{c}\lambda \text { Coverage } \\
(\AA)\end{array}$ \\
& & & & \\
\hline IRAS F22456-5125 & 2007 August 9 & 2400 & 4.3 & $3667-5409$ \\
IRAS F22456-5125 & 2007 August 16 & 2400 & 3.1 & $5639-6947$ \\
MR 2251-178 & 2007 August 15 & 2400 & 4.3 & $3667-5423$ \\
MR 2251-178 & 2007 August 17 & 2400 & 3.1 & $5639-6947$ \\
WPVS 007 & 2007 August 15 & 2400 & 4.3 & $3667-5423$ \\
WPVS 007 & 2007 August 17 & 1800 & 3.1 & $5638-6947$ \\
\hline
\end{tabular}


Table 2. AGN Continuum Levels

\begin{tabular}{|c|c|c|c|c|c|c|}
\hline Object & Observation ID & $\begin{array}{c}\text { Julian Date } \\
+2400000 \text { days }\end{array}$ & $\begin{array}{c}\text { Flux } \\
\times 10^{14} \\
\operatorname{ergs~s}^{-1} \AA^{-1}\end{array}$ & $\begin{array}{l}\operatorname{Bin} \\
(\AA)\end{array}$ & $\begin{array}{c}\log L_{\lambda} \\
\operatorname{ergs~s}^{-1} \AA^{-1}\end{array}$ & $\begin{array}{c}\sigma_{\log L_{\lambda}} \\
\operatorname{ergs~s}^{-1} \AA \AA^{-1}\end{array}$ \\
\hline WPVS 007 & D8060201000 & 52950.360 & 0.48 & 1020 & 39.88 & 0.11 \\
\hline \multirow[t]{8}{*}{ QSO0045+3926 } & Z0020401000 & 51874.176 & 1.01 & 1110 & 41.54 & 0.03 \\
\hline & D1310101000 & 52921.336 & 1.34 & 1110 & 41.66 & 0.05 \\
\hline & D1310102000 & 52922.707 & 1.40 & 1110 & 41.68 & 0.05 \\
\hline & D1310103000 & 52924.391 & 1.14 & 1110 & 41.60 & 0.04 \\
\hline & D1310104000 & 52983.242 & 0.88 & 1110 & 41.48 & 0.06 \\
\hline & D1310105000 & 53335.281 & 1.28 & 1110 & 41.65 & 0.07 \\
\hline & D1310106000 & 53336.105 & 1.24 & 1110 & 41.63 & 0.07 \\
\hline & D1310107000 & 53337.000 & 1.23 & 1110 & 41.63 & 0.07 \\
\hline \multirow[t]{2}{*}{ TONS180 } & P1010502000 & 51524.742 & 6.16 & 1160 & 41.66 & 0.05 \\
\hline & D0280101000 & 53199.973 & 4.49 & 1160 & 41.52 & 0.05 \\
\hline MRK1044 & D0410101000 & 53006.246 & 2.52 & 1020 & 40.12 & 0.09 \\
\hline NGC985 & P1010903000 & 51536.551 & 2.45 & 1020 & 40.94 & 0.05 \\
\hline EUVEJ0349-537 & E8970301000 & 53237.559 & 0.36 & 1110 & 41.06 & 0.04 \\
\hline IRASF04250-5718 & D8080801000 & 52886.609 & 3.66 & 1110 & 41.89 & 0.07 \\
\hline \multirow[t]{3}{*}{ MRK79 } & P1011701000 & 51512.949 & 1.15 & 1110 & 40.04 & 0.11 \\
\hline & P1011702000 & 51558.152 & 1.13 & 1110 & 40.03 & 0.10 \\
\hline & P1011703000 & 51596.613 & 1.41 & 1110 & 40.12 & 0.08 \\
\hline MRK10 & Z9072801000 & 52673.195 & 1.07 & 1110 & 40.24 & 0.11 \\
\hline IR07546+3928 & S6011801000 & 52317.062 & 0.11 & 1160 & 40.29 & 0.08 \\
\hline \multirow[t]{4}{*}{ PG0804+761 } & P1011901000 & 51456.504 & 7.84 & 1160 & 42.18 & 0.01 \\
\hline & P1011903000 & 54548.312 & 14.08 & 1160 & 42.44 & 0.02 \\
\hline & S6011001000 & 52311.059 & 5.72 & 1160 & 42.05 & 0.02 \\
\hline & S6011002000 & 52315.406 & 5.90 & 1160 & 42.06 & 0.02 \\
\hline \multirow[t]{3}{*}{ Ton951 } & P1012002000 & 53080.004 & 4.62 & 1160 & 41.56 & 0.06 \\
\hline & D0280304000 & 51595.316 & 3.42 & 1160 & 41.43 & 0.03 \\
\hline & D0280301000 & 53079.102 & 2.90 & 1160 & 41.36 & 0.07 \\
\hline \multirow[t]{3}{*}{ IRAS09149-62 } & A0020503000 & 51581.129 & 1.42 & 1110 & 40.95 & 0.04 \\
\hline & S7011003000 & 53461.098 & 0.80 & 1110 & 40.70 & 0.09 \\
\hline & U1072202000 & 53929.031 & 0.74 & 1110 & 40.67 & 0.06 \\
\hline MKN141 & D8061001000 & 53085.585 & 0.07 & 1110 & 39.09 & 0.38 \\
\hline \multirow[t]{3}{*}{ NGC3516 } & P1110404000 & 51651.957 & 1.24 & 1020 & 38.97 & 0.18 \\
\hline & G9170101000 & 53776.217 & 0.69 & 1020 & 38.71 & 0.20 \\
\hline & G9170102000 & 54123.776 & 0.63 & 1020 & 38.67 & 0.23 \\
\hline \multirow[t]{4}{*}{ ESO265-G23 } & A1210405000 & 51978.219 & 0.38 & 1110 & 40.36 & 0.13 \\
\hline & A1210407000 & 52341.223 & 0.39 & 1110 & 40.37 & 0.07 \\
\hline & A1210408000 & 52393.352 & 0.73 & 1110 & 40.64 & 0.03 \\
\hline & A1210409000 & 52394.416 & 1.03 & 1110 & 40.79 & 0.04 \\
\hline \multirow[t]{5}{*}{ NGC3783 } & P1013301000 & 51576.621 & 4.90 & 1160 & 39.95 & 0.03 \\
\hline & B1070102000 & 51969.199 & 4.94 & 1160 & 39.95 & 0.03 \\
\hline & B1070103000 & 51979.535 & 3.49 & 1160 & 39.80 & 0.03 \\
\hline & B1070104000 & 51999.453 & 5.81 & 1160 & 40.02 & 0.03 \\
\hline & B1070105000 & 52087.727 & 4.47 & 1160 & 39.91 & 0.04 \\
\hline
\end{tabular}


Table 2-Continued

\begin{tabular}{|c|c|c|c|c|c|c|}
\hline Object & Observation ID & $\begin{array}{c}\text { Julian Date } \\
+2400000 \text { days }\end{array}$ & $\begin{array}{c}\text { Flux } \\
\times 10^{14} \\
\operatorname{ergs~s}^{-1} \AA^{-1}\end{array}$ & $\begin{array}{l}\text { Bin } \\
(\AA)\end{array}$ & $\begin{array}{c}\log \mathrm{L}_{\lambda} \\
\operatorname{ergs~s}^{-1} \AA^{-1}\end{array}$ & $\begin{array}{c}\sigma_{\log L_{\lambda}} \\
\operatorname{ergs~s}^{-1} \AA \AA^{-1}\end{array}$ \\
\hline & B1070106000 & 51975.855 & 2.87 & 1160 & 39.72 & 0.03 \\
\hline & E0310101000 & 53130.965 & 4.11 & 1160 & 39.87 & 0.03 \\
\hline \multirow[t]{3}{*}{ NGC4051 } & B0620201000 & 52362.582 & 1.29 & 1020 & 38.13 & 0.05 \\
\hline & C0190101000 & 52657.988 & 1.35 & 1020 & 38.15 & 0.07 \\
\hline & C0190102000 & 52717.824 & 1.51 & 1020 & 38.20 & 0.07 \\
\hline \multirow[t]{4}{*}{ NGC4151 } & P1110505000 & 51609.410 & 2.50 & 1020 & 38.72 & 0.05 \\
\hline & P2110201000 & 52008.199 & 1.71 & 1020 & 38.56 & 0.08 \\
\hline & C0920101000 & 52423.469 & 21.52 & 1020 & 39.66 & 0.03 \\
\hline & P2110202000 & 52427.199 & 12.67 & 1020 & 39.43 & 0.05 \\
\hline RXJ1230.8+0115 & P1019001000 & 51715.832 & 5.24 & 1110 & 42.14 & 0.06 \\
\hline TOL1238-364 & D0100101000 & 51945.742 & 1.47 & 1110 & 39.53 & 0.06 \\
\hline \multirow[t]{2}{*}{$\mathrm{PG} 1351+640$} & P1072501000 & 51562.117 & 1.19 & 1160 & 41.25 & 0.03 \\
\hline & S6010701000 & 52306.922 & 1.68 & 1160 & 41.40 & 0.02 \\
\hline \multirow{7}{*}{ MRK279 } & P1080303000 & 51540.527 & 11.45 & 1110 & 41.31 & 0.01 \\
\hline & P1080304000 & 51554.562 & 9.61 & 1110 & 41.23 & 0.02 \\
\hline & C0900201000 & 52413.258 & 0.61 & 1110 & 40.04 & 0.02 \\
\hline & D1540101000 & 52772.410 & 9.20 & 1110 & 41.21 & 0.01 \\
\hline & F3250103000 & 53711.961 & 7.35 & 1110 & 41.12 & 0.02 \\
\hline & F3250104000 & 53713.277 & 2.86 & 1110 & 40.70 & 0.08 \\
\hline & F3250106000 & 53769.598 & 4.53 & 1110 & 40.90 & 0.05 \\
\hline RXJ135515+561244 & D8061601000 & 52712.387 & 0.39 & 1110 & 41.04 & 0.02 \\
\hline PG1404+226 & P2100401000 & 52071.652 & 0.93 & 1110 & 41.23 & 0.10 \\
\hline PG1411+442 & A0601010000 & 51675.637 & 2.01 & 1160 & 41.49 & 0.06 \\
\hline NGC5548 & P1014601000 & 51703.055 & 1.55 & 1020 & 39.94 & 0.05 \\
\hline \multirow[t]{4}{*}{ MRK817 } & P1080401000 & 51591.965 & 9.05 & 1110 & 41.23 & 0.03 \\
\hline & P1080402000 & 51592.766 & 7.20 & 1110 & 41.13 & 0.03 \\
\hline & P1080403000 & 51902.176 & 3.89 & 1110 & 40.87 & 0.003 \\
\hline & P1080404000 & 51958.891 & 6.25 & 1110 & 41.07 & 0.01 \\
\hline \multirow[t]{3}{*}{ MRK290 } & P1072901000 & 51620.230 & 0.45 & 1110 & 39.87 & 0.08 \\
\hline & D0760101000 & 52819.457 & 3.44 & 1110 & 40.76 & 0.06 \\
\hline & E0840101000 & 53171.730 & 3.83 & 1110 & 40.81 & 0.06 \\
\hline \multirow[t]{3}{*}{ MRK876 } & P1073101000 & 51467.766 & 0.24 & 1110 & 40.88 & 0.03 \\
\hline & D0280201000 & 52776.450 & 5.77 & 1110 & 42.26 & 0.08 \\
\hline & D0280203000 & 53049.797 & 5.71 & 1110 & 42.26 & 0.03 \\
\hline \multirow{3}{*}{ MRK509 } & X0170101000 & 51485.445 & 7.41 & 1020 & 41.23 & 0.05 \\
\hline & X0170102000 & 51488.703 & 8.00 & 1020 & 41.26 & 0.06 \\
\hline & P1080601000 & 51792.750 & 9.14 & 1020 & 41.32 & 0.05 \\
\hline \multirow[t]{4}{*}{ IIZW136 } & P1018301000 & 51863.473 & 2.36 & 1160 & 41.25 & 0.04 \\
\hline & P1018302000 & 53152.684 & 3.72 & 1160 & 41.45 & 0.07 \\
\hline & P1018303000 & 53153.727 & 2.79 & 1160 & 41.33 & 0.07 \\
\hline & P1018304000 & 53310.586 & 3.71 & 1160 & 41.45 & 0.04 \\
\hline AKN564 & B0620101000 & 52089.816 & 1.03 & 1110 & 40.08 & 0.02 \\
\hline IRAS F22456-5125 & Z9073901000 & 52541.902 & 1.12 & 1110 & 41.33 & 0.10 \\
\hline
\end{tabular}


Table 2-Continued

\begin{tabular}{ccccccc}
\hline \hline Object & Observation ID & $\begin{array}{c}\text { Julian Date } \\
+2400000 \text { days }\end{array}$ & $\begin{array}{c}\text { Flux } \\
\times 10^{14} \\
\operatorname{ergs~s}^{-1} \AA^{-1}\end{array}$ & $\begin{array}{c}\text { Bin } \\
(\AA)\end{array}$ & $\begin{array}{c}\text { Log } L_{\lambda} \\
\operatorname{ergs~s}^{-1} \AA^{-1}\end{array}$ & $\begin{array}{c}\sigma_{L_{\text {Log }} L_{\lambda}} \\
\operatorname{ergs~s}^{-1} \AA^{-1}\end{array}$ \\
\hline \multirow{2}{*}{ MR 2251-178 } & Z9073902000 & 52542.457 & 1.75 & 1110 & 41.52 & 0.04 \\
NGC7469 & P1111010000 & 53194.629 & 6.08 & 1110 & 42.07 & 0.08 \\
& P1074101000 & 51724.101 & 1.30 & 1020 & 39.52 & 0.08 \\
& C0900101000 & 52621.797 & 2.11 & 1020 & 39.73 & 0.14 \\
\hline
\end{tabular}


Table 3. Measured Quantities

\begin{tabular}{|c|c|c|c|c|c|c|c|c|c|c|c|c|c|}
\hline \multirow[t]{2}{*}{ Object } & \multirow[t]{2}{*}{ Obs ID } & \multirow[t]{2}{*}{ Comp } & \multicolumn{2}{|c|}{ Equivalent } & \multirow{2}{*}{$\begin{array}{c}\text { Width } \\
\text { O VIr } \\
(\AA)\end{array}$} & \multicolumn{3}{|c|}{$\sigma_{E W}$} & \multicolumn{3}{|c|}{ FWHM } & \multirow{2}{*}{$\begin{array}{c}\mathbf{V}_{r} \\
\left(\mathrm{~km} \mathrm{~s}^{-1}\right)\end{array}$} & \multirow{2}{*}{$\begin{array}{c}\sigma_{v e l} \\
\left(\mathrm{~km} \mathrm{~s}^{-1}\right.\end{array}$} \\
\hline & & & $\begin{array}{l}\mathrm{Ly} \beta \\
(\AA)\end{array}$ & $\begin{array}{l}\text { O VIb } \\
(\AA)\end{array}$ & & $\begin{array}{l}\mathrm{Ly} \beta \\
(\AA)\end{array}$ & $\begin{array}{l}\text { O VIb } \\
(\AA)\end{array}$ & $\begin{array}{c}\text { O VIr } \\
(\AA)\end{array}$ & $\operatorname{Ly} \beta$ & $\begin{array}{l}\mathrm{O} \text { VIb } \\
\left(\mathrm{km} \mathrm{s}^{-1}\right)\end{array}$ & O VIr & & \\
\hline WPVS 007 & D8060201000 & 1 & 1.91 & 2.06 & 1.76 & 0.14 & 0.13 & 0.14 & 714 & 639 & 615 & -397 & 26 \\
\hline \multirow[t]{8}{*}{ QSO0045+3926 } & Z0020401000 & 1 & 0.29 & 0.61 & 0.29 & 0.04 & 0.06 & 0.05 & 100 & 172 & 69 & 361 & 13 \\
\hline & D1310101000 & 1 & 0.19 & 0.45 & 0.38 & 0.01 & 0.01 & 0.01 & 56 & 128 & 98 & 351 & 5 \\
\hline & D1310102000 & 1 & 0.19 & 0.38 & 0.29 & 0.02 & 0.02 & 0.02 & 69 & 101 & 107 & 345 & 12 \\
\hline & D1310103000 & 1 & 0.29 & 0.41 & 0.27 & 0.08 & 0.06 & 0.06 & 77 & 121 & 63 & 340 & 22 \\
\hline & D1310104000 & 1 & 0.17 & 0.36 & 0.31 & 0.01 & 0.02 & 0.02 & 82 & 107 & 74 & 346 & 16 \\
\hline & D1310105000 & 1 & 0.13 & 0.40 & 0.33 & 0.02 & 0.02 & 0.02 & 86 & 107 & 94 & 371 & 6 \\
\hline & D1310106000 & 1 & 0.16 & 0.42 & 0.28 & 0.02 & 0.02 & 0.02 & 69 & 91 & 85 & 375 & 7 \\
\hline & D1310107000 & 1 & 0.14 & 0.33 & 0.32 & 0.02 & 0.03 & 0.02 & 60 & 77 & 73 & 396 & 9 \\
\hline \multirow[t]{2}{*}{ TONS180 } & P1010502000 & 1 & & & 0.13 & & & 0.03 & & & 65 & -1732 & \\
\hline & D0280101000 & 1 & & & 0.17 & & & 0.03 & & & 85 & -1792 & \\
\hline \multirow{5}{*}{$\begin{array}{r}\text { MRK1044 } \\
\text { NGC985 }\end{array}$} & D0410101000 & 1 & 0.20 & 0.22 & & 0.04 & 0.03 & & 65 & 92 & & -1110 & 2 \\
\hline & P1010903000 & 1 & & 0.11 & 0.06 & & 0.01 & 0.01 & & 96 & 77 & -814 & 20 \\
\hline & P1010903000 & 2 & 0.12 & 0.26 & 0.20 & 0.02 & 0.01 & 0.01 & 34 & 72 & 55 & -678 & 6 \\
\hline & P1010903000 & 3 & 0.56 & 0.71 & 0.65 & 0.02 & 0.02 & 0.01 & 158 & 199 & 180 & -454 & 51 \\
\hline & P1010903000 & 4 & 0.15 & 0.16 & 0.23 & 0.02 & 0.01 & 0.01 & 41 & 44 & 63 & -268 & 17 \\
\hline EUVEJ0349-537 & E8970301000 & 1 & 0.47 & 0.71 & 0.60 & 0.04 & 0.04 & 0.06 & 128 & 218 & 165 & 26 & 21 \\
\hline \multirow[t]{2}{*}{ IRASF04250-5718 } & D8080801000 & 1 & 0.56 & 0.62 & 0.64 & 0.06 & 0.07 & 0.07 & 159 & 195 & 184 & -216 & 5 \\
\hline & D8080801000 & 2 & 0.15 & 0.25 & 0.20 & 0.06 & 0.03 & 0.03 & 41 & 66 & 51 & -64 & 2 \\
\hline \multirow[t]{6}{*}{ MRK79 } & P1011701000 & 1 & 0.35 & 0.65 & 0.76 & 0.18 & 0.17 & 0.17 & 117 & 242 & 220 & -350 & 12 \\
\hline & P1011702000 & 1 & 0.30 & 0.52 & 0.43 & 0.10 & 0.09 & 0.08 & 175 & 240 & 160 & -325 & 35 \\
\hline & P1011703000 & 1 & 0.18 & 0.57 & 0.52 & 0.07 & 0.08 & 0.06 & 86 & 198 & 194 & -326 & 27 \\
\hline & P1011701000 & 2 & & & 0.39 & & & 0.13 & & & 255 & -1404 & \\
\hline & P1011702000 & 2 & & & 0.31 & & & 0.08 & & & 87 & -1387 & \\
\hline & P1011703000 & 2 & & & 0.28 & & & 0.07 & & & 81 & -1367 & \\
\hline MRK10 & Z9072801000 & 1 & 1.03 & & 0.92 & 0.09 & & 0.13 & 344 & & 227 & -126 & 18 \\
\hline \multirow[t]{2}{*}{ IR07546+3928 } & S6011801000 & 1 & 1.07 & 1.49 & 1.00 & 0.05 & 0.03 & 0.02 & 280 & 436 & 351 & -1777 & 9 \\
\hline & S6011801000 & 2 & 0.98 & 1.48 & 1.37 & 0.03 & 0.03 & 0.03 & 263 & 395 & 361 & -1116 & 12 \\
\hline \multirow[t]{4}{*}{ PG0804+761 } & P1011901000 & 1 & 0.21 & 0.13 & 0.09 & 0.004 & 0.003 & 0.003 & 69 & 67 & 64 & 363 & 7 \\
\hline & P1011903000 & 1 & 0.23 & 0.14 & 0.06 & 0.01 & 0.01 & 0.01 & 75 & 66 & 55 & 370 & 2 \\
\hline & S6011001000 & 1 & 0.19 & 0.11 & 0.10 & 0.01 & 0.004 & 0.01 & 63 & 64 & 81 & 356 & 1 \\
\hline & S6011002000 & 1 & 0.20 & 0.11 & 0.09 & 0.01 & 0.01 & 0.01 & 74 & 66 & 71 & 358 & 5 \\
\hline
\end{tabular}


Table 3-Continued

\begin{tabular}{|c|c|c|c|c|c|c|c|c|c|c|c|c|c|}
\hline \multirow[t]{2}{*}{ Object } & \multirow{2}{*}{ Obs ID } & \multirow[t]{2}{*}{ Comp } & \multicolumn{2}{|c|}{ Equivalent } & \multirow{2}{*}{$\begin{array}{c}\text { Width } \\
\text { O VIr } \\
(\AA)\end{array}$} & \multicolumn{3}{|c|}{$\sigma_{E W}$} & \multicolumn{3}{|c|}{ FWHM } & \multirow{2}{*}{$\begin{array}{c}\mathbf{V}_{r} \\
\left(\mathrm{~km} \mathrm{~s}^{-1}\right)\end{array}$} & \multirow{2}{*}{$\begin{array}{c}\sigma_{v e l} \\
\left(\mathrm{~km} \mathrm{~s}^{-1}\right)\end{array}$} \\
\hline & & & $\begin{array}{l}\mathrm{Ly} \beta \\
(\AA)\end{array}$ & $\begin{array}{l}\text { O VIb } \\
(\AA)\end{array}$ & & $\begin{array}{l}\mathrm{Ly} \beta \\
(\AA)\end{array}$ & $\begin{array}{c}\text { O VIb } \\
(\AA)\end{array}$ & $\begin{array}{l}\text { O VIr } \\
(\AA)\end{array}$ & $\operatorname{Ly} \beta$ & $\begin{array}{c}\mathrm{O} \text { VIb } \\
\left(\mathrm{km} \mathrm{s}^{-1}\right)\end{array}$ & O VIr & & \\
\hline \multirow{6}{*}{ IRAS09149-62 } & P1012002000 & 1 & 0.60 & 0.43 & 0.43 & 0.02 & 0.01 & 0.01 & 159 & 123 & 118 & 179 & 16 \\
\hline & D0280304000 & 1 & 0.69 & 0.42 & 0.46 & 0.05 & 0.03 & 0.02 & 190 & 150 & 128 & 173 & 25 \\
\hline & D0280301000 & 1 & 0.66 & 0.52 & 0.41 & 0.05 & 0.05 & 0.02 & 186 & 149 & 114 & 149 & 23 \\
\hline & A0020503000 & 1 & & 1.72 & 1.26 & & 0.22 & 0.12 & & 504 & 389 & 18 & 18 \\
\hline & S7011003000 & 1 & & & 1.08 & & & 0.11 & & & 377 & 31 & \\
\hline & U1072202000 & 1 & & & 1.33 & & & 0.08 & & & 431 & 56 & \\
\hline \multirow[t]{12}{*}{ NGC3516 } & P1110404000 & 1 & 0.27 & 1.76 & 0.06 & 0.07 & 0.09 & 0.03 & 168 & 510 & 52 & -1511 & 360 \\
\hline & G9170101000 & 1 & 0.33 & 1.40 & 0.14 & 0.13 & 0.17 & 0.20 & 177 & 468 & 133 & -1362 & 6.8 \\
\hline & G9170102000 & 1 & 0.23 & 0.53 & 0.15 & 0.07 & 0.07 & 0.04 & 133 & 202 & 63 & -1343 & 101 \\
\hline & P1110404000 & 2 & & 0.54 & 0.08 & & 0.06 & 0.05 & & 250 & 162 & -866 & 125 \\
\hline & G9170101000 & 2 & 0.38 & 0.57 & 0.64 & 0.16 & 0.12 & 0.20 & 212 & 239 & 331 & -888 & 38 \\
\hline & G9170102000 & 2 & 0.15 & 0.28 & 0.34 & 0.06 & 0.05 & 0.05 & 112 & 99 & 198 & -911 & 52 \\
\hline & P1110404000 & 3 & & 0.28 & 0.35 & & 0.04 & 0.03 & & 123 & 46 & -410 & 15 \\
\hline & G9170101000 & 3 & 0.38 & & 0.58 & 0.15 & & 0.12 & 168 & & 196 & -460 & 10 \\
\hline & G9170102000 & 3 & 0.34 & 0.32 & 0.38 & 0.06 & 0.06 & 0.05 & 113 & 98 & 100 & -456 & 2 \\
\hline & P1110404000 & 4 & 0.38 & 0.38 & 0.40 & 0.08 & 0.05 & 0.06 & 110 & 163 & 239 & -216 & 1 \\
\hline & G9170101000 & 4 & 0.58 & & 0.46 & 0.17 & & 0.23 & 226 & & 174 & -236 & 30 \\
\hline & G9170102000 & 4 & 0.67 & 0.79 & 0.80 & 0.06 & 0.06 & 0.07 & 233 & 287 & 302 & -227 & 6 \\
\hline \multirow[t]{4}{*}{ ESO265-G23 } & A1210405000 & 1 & & 0.29 & 0.15 & & 0.15 & 0.09 & & 127 & 126 & -178 & 13 \\
\hline & A1210407000 & 1 & & 0.34 & 0.32 & & 0.05 & 0.04 & & 141 & 73 & -157 & 5 \\
\hline & A1210408000 & 1 & & 0.29 & 0.36 & & 0.10 & 0.08 & & 63 & 288 & -124 & 14 \\
\hline & A1210409000 & 1 & & 0.34 & 0.30 & & 0.13 & 0.10 & & 92 & 81 & -157 & 26 \\
\hline \multirow[t]{7}{*}{ NGC3783 } & P1013301000 & 1 & 1.03 & 1.19 & 0.78 & 0.02 & 0.02 & 0.03 & 403 & 463 & 404 & -660 & 10 \\
\hline & B1070102000 & 1 & 1.12 & 1.18 & 0.62 & 0.03 & 0.02 & 0.03 & 394 & 467 & 456 & -668 & 20 \\
\hline & B1070103000 & 1 & 1.11 & 1.19 & 0.70 & 0.04 & 0.03 & 0.03 & 400 & 452 & 449 & -663 & 16 \\
\hline & B1070104000 & 1 & 0.99 & 1.12 & 0.78 & 0.03 & 0.03 & 0.02 & 332 & 443 & 393 & -675 & 19 \\
\hline & B1070105000 & 1 & 0.88 & 1.16 & 0.65 & 0.03 & 0.03 & 0.03 & 377 & 459 & 404 & -682 & 13 \\
\hline & B1070106000 & 1 & 1.17 & 1.20 & 0.76 & 0.04 & 0.03 & 0.03 & 427 & 457 & 395 & -664 & 12 \\
\hline & E0310101000 & 1 & 1.05 & 1.30 & 0.80 & 0.02 & 0.03 & 0.02 & 380 & 467 & 371 & -657 & 30 \\
\hline \multirow[t]{3}{*}{ NGC4051 } & B0620201000 & 1 & & 2.28 & & & 0.00 & & & 672 & & -374 & \\
\hline & C0190101000 & 1 & & 2.13 & & & 0.04 & & & 696 & & -348 & \\
\hline & C0190102000 & 1 & & 2.14 & 1.88 & & 0.05 & 0.04 & & 670 & 627 & -359 & 12 \\
\hline
\end{tabular}


Table 3-Continued

\begin{tabular}{|c|c|c|c|c|c|c|c|c|c|c|c|c|c|}
\hline \multirow[t]{2}{*}{ Object } & \multirow[t]{2}{*}{ Obs ID } & \multirow[t]{2}{*}{ Comp } & \multicolumn{2}{|c|}{ Equivalent } & \multirow{2}{*}{$\begin{array}{c}\text { Width } \\
\text { O VIr } \\
(\AA)\end{array}$} & \multicolumn{3}{|c|}{$\sigma_{E W}$} & \multicolumn{3}{|c|}{ FWHM } & \multirow{2}{*}{$\begin{array}{c}\mathbf{V}_{r} \\
\left(\mathrm{~km} \mathrm{~s}^{-1}\right)\end{array}$} & \multirow{2}{*}{$\begin{array}{c}\sigma_{v e l} \\
\left(\mathrm{~km} \mathrm{~s}^{-1}\right)\end{array}$} \\
\hline & & & $\begin{array}{l}\mathrm{Ly} \beta \\
(\AA)\end{array}$ & $\begin{array}{c}\text { O VIb } \\
(\AA)\end{array}$ & & $\begin{array}{l}\mathrm{Ly} \beta \\
(\AA)\end{array}$ & $\begin{array}{c}\text { O VIb } \\
(\AA)\end{array}$ & $\begin{array}{l}\text { O VIr } \\
(\AA)\end{array}$ & $\operatorname{Ly} \beta$ & $\begin{array}{c}\text { O VIb } \\
\left(\mathrm{km} \mathrm{s}^{-1}\right)\end{array}$ & O VIr & & \\
\hline \multirow[t]{8}{*}{ NGC4151 } & P1110505000 & 1 & & 0.91 & & & 0.04 & & & 473 & & -706 & \\
\hline & P2110201000 & 1 & & 1.48 & 1.21 & & 0.07 & 0.04 & & 514 & 522 & -574 & 222 \\
\hline & C0920101000 & 1 & & 1.77 & 1.95 & & 0.01 & 0.01 & & 718 & 681 & -397 & 41 \\
\hline & P2110202000 & 1 & & 1.66 & 1.99 & & 0.08 & 0.05 & & 662 & 716 & -413 & 56 \\
\hline & P1019001000 & 1 & & 1.90 & 1.57 & & 0.09 & 0.08 & & 542 & 476 & -2994 & 16 \\
\hline & P1019001000 & 2 & & 0.27 & 0.26 & & 0.06 & 0.05 & & 71 & 69 & -2420 & 50 \\
\hline & P1019001000 & 3 & & 1.19 & 1.07 & & 0.08 & 0.06 & & 311 & 278 & -2014 & 3 \\
\hline & P1019001000 & 4 & & 1.17 & 1.08 & & 0.06 & 0.08 & & 304 & 280 & 120 & 2 \\
\hline TOL1238-364 & D0100101000 & 1 & & 1.10 & 1.51 & & 0.14 & 0.15 & & 339 & 454 & -252 & 75 \\
\hline \multirow[t]{4}{*}{ PG1351+640 } & P1072501000 & 1 & 0.60 & 1.31 & 1.20 & 0.01 & 0.01 & 0.01 & 182 & 453 & 447 & -1705 & 73 \\
\hline & S6010701000 & 1 & 0.54 & 1.37 & 1.42 & 0.02 & 0.02 & 0.02 & 208 & 455 & 429 & -1715 & 60 \\
\hline & P1072501000 & 2 & 1.51 & 2.08 & 1.94 & 0.02 & 0.01 & 0.01 & 407 & 559 & 516 & -883 & 24 \\
\hline & S6010701000 & 2 & 1.23 & 2.16 & 2.12 & 0.02 & 0.02 & 0.02 & 333 & 579 & 565 & -899 & 11 \\
\hline \multirow[t]{7}{*}{ MRK279 } & P1080303000 & 1 & 0.77 & 1.20 & 0.75 & 0.01 & 0.01 & 0.01 & 321 & 478 & 301 & -312 & 6 \\
\hline & P1080304000 & 1 & 0.82 & 1.15 & 0.77 & 0.02 & 0.02 & 0.02 & 328 & 482 & 307 & -328 & 4 \\
\hline & $\mathrm{C} 0900201000$ & 1 & 1.01 & 0.96 & 1.06 & 0.07 & 0.06 & 0.06 & 299 & 497 & 312 & -351 & 2 \\
\hline & D1540101000 & 1 & 0.76 & 1.32 & 0.97 & 0.01 & 0.01 & 0.01 & 290 & 474 & 319 & -308 & 8 \\
\hline & F3250103000 & 1 & 1.19 & 1.80 & 0.97 & 0.13 & 0.10 & 0.10 & 285 & 508 & 306 & -404 & 33 \\
\hline & F3250104000 & 1 & 0.87 & 1.64 & 0.93 & 0.13 & 0.13 & 0.11 & 392 & 518 & 366 & -302 & 12 \\
\hline & F3250106000 & 1 & 0.69 & 1.26 & 0.90 & 0.22 & 0.21 & 0.16 & 324 & 502 & 328 & -308 & 28 \\
\hline \multirow[t]{2}{*}{ RXJ135515+561244 } & D8061601000 & 1 & 0.34 & 0.61 & 0.76 & 0.04 & 0.04 & 0.05 & 86 & 160 & 205 & -834 & 12 \\
\hline & D8061601000 & 2 & & 0.69 & 0.48 & & 0.05 & 0.03 & & 180 & 186 & -163 & 28 \\
\hline \multirow[t]{2}{*}{ PG1404+226 } & P2100401000 & 1 & 0.19 & 0.54 & 0.36 & 0.04 & 0.03 & 0.04 & 88 & 151 & 127 & -166 & 57 \\
\hline & P2100401000 & 2 & & 1.47 & 1.11 & & 0.06 & 0.07 & & 330 & 284 & 134 & 17 \\
\hline PG1411+442 & A0601010000 & 1 & & & 0.64 & & & 0.03 & & & 170 & 55 & \\
\hline \multirow[t]{2}{*}{ NGC5548 } & P1014601000 & 1 & & 0.35 & 0.25 & & 0.02 & 0.02 & & 161 & 195 & -441 & 4 \\
\hline & P1014601000 & 2 & & 0.25 & 0.31 & & 0.02 & 0.02 & & 111 & 142 & -683 & 5 \\
\hline \multirow[t]{5}{*}{ MRK817 } & P1080401000 & 1 & 0.08 & 0.60 & 0.41 & 0.05 & 0.04 & 0.05 & 319 & 207 & 143 & -4198 & 5 \\
\hline & P1080402000 & 1 & 0.12 & 0.51 & 0.39 & 0.05 & 0.05 & 0.06 & 236 & 237 & 146 & -4184 & 16 \\
\hline & P1080403000 & 1 & 0.10 & 0.41 & 0.36 & 0.01 & 0.02 & 0.02 & 134 & 273 & 265 & -4166 & 32 \\
\hline & P1080404000 & 1 & 0.06 & 0.40 & 0.33 & 0.01 & 0.00 & 0.01 & 210 & 208 & 154 & -4144 & 12 \\
\hline & P1080404000 & 2 & & 0.12 & 0.05 & & 0.01 & 0.01 & & 243 & 142 & -3680 & 27 \\
\hline
\end{tabular}


Table 3-Continued

\begin{tabular}{|c|c|c|c|c|c|c|c|c|c|c|c|c|c|}
\hline \multirow[t]{2}{*}{ Object } & \multirow[t]{2}{*}{ Obs ID } & \multirow[t]{2}{*}{ Comp } & \multicolumn{2}{|c|}{ Equivalent } & \multirow{2}{*}{$\begin{array}{c}\text { Width } \\
\text { O VIr } \\
(\AA)\end{array}$} & \multicolumn{3}{|c|}{$\sigma_{E W}$} & \multicolumn{3}{|c|}{ FWHM } & \multirow{2}{*}{$\begin{array}{c}\mathbf{V}_{r} \\
\left(\mathrm{~km} \mathrm{~s}^{-1}\right)\end{array}$} & \multirow{2}{*}{$\begin{array}{c}\sigma_{v e l} \\
\left(\mathrm{~km} \mathrm{~s}^{-1}\right.\end{array}$} \\
\hline & & & $\begin{array}{l}\mathrm{Ly} \beta \\
(\AA)\end{array}$ & $\begin{array}{c}\text { O VIb } \\
(\AA)\end{array}$ & & $\begin{array}{l}\mathrm{Ly} \beta \\
(\AA)\end{array}$ & $\begin{array}{c}\text { O VIb } \\
(\AA)\end{array}$ & $\begin{array}{c}\text { O VIr } \\
(\AA)\end{array}$ & $\operatorname{Ly} \beta$ & $\begin{array}{c}\text { O VIb } \\
\left(\mathrm{km} \mathrm{s}^{-1}\right)\end{array}$ & O VIr & & \\
\hline \multirow{4}{*}{ MRK290 } & P1080404000 & 3 & & 0.12 & 0.09 & & 0.01 & 0.01 & & 118 & 139 & -2966 & 6 \\
\hline & P1072901000 & 1 & 0.71 & 0.62 & 0.54 & 0.12 & 0.10 & 0.08 & 185 & 190 & 142 & -225 & 14 \\
\hline & D0760101000 & 1 & 0.51 & 0.59 & 0.48 & 0.07 & 0.05 & 0.05 & 141 & 192 & 165 & -212 & 17 \\
\hline & E0840101000 & 1 & 0.54 & 0.48 & 0.43 & 0.05 & 0.05 & 0.05 & 180 & 157 & 158 & -187 & 72 \\
\hline \multirow[t]{2}{*}{ MRK876 } & P1073101000 & 1 & & 0.11 & 0.09 & & 0.01 & 0.01 & & 73 & 99 & -3735 & 3 \\
\hline & D0280203000 & 1 & & 0.15 & 0.11 & & 0.00 & 0.00 & & 101 & 94 & -3724 & 0.8 \\
\hline \multirow[t]{6}{*}{ MRK509 } & X0170101000 & 1 & 0.55 & 0.70 & 0.52 & 0.02 & 0.01 & 0.01 & 217 & 217 & 182 & -305 & 16 \\
\hline & X0170102000 & 1 & 0.65 & 0.80 & 0.58 & 0.03 & 0.02 & 0.02 & 218 & 204 & 190 & -304 & 19 \\
\hline & P1080601000 & 1 & 0.61 & 0.67 & 0.56 & 0.01 & 0.01 & 0.01 & 170 & 201 & 177 & -300 & 18 \\
\hline & X0170101000 & 2 & 0.95 & 1.23 & 1.12 & 0.02 & 0.01 & 0.01 & 303 & 329 & 346 & 103 & 12 \\
\hline & X0170102000 & 2 & 1.05 & 1.27 & 1.16 & 0.03 & 0.02 & 0.03 & 321 & 331 & 322 & 27 & 11 \\
\hline & P1080601000 & 2 & 1.15 & 1.15 & 1.19 & 0.01 & 0.01 & 0.01 & 330 & 333 & 341 & 90 & 12 \\
\hline \multirow[t]{8}{*}{ IIZW136 } & P1018301000 & 1 & & 0.42 & & & 0.02 & & & 146 & & -1487 & \\
\hline & P1018302000 & 1 & & 0.47 & & & 0.03 & & & 173 & & -1512 & \\
\hline & P1018303000 & 1 & & 0.49 & & & 0.04 & & & 96 & & -1517 & \\
\hline & P1018304000 & 1 & & 0.45 & & & 0.03 & & & 167 & & -1512 & \\
\hline & P1018301000 & 2 & & & 0.28 & & & 0.02 & & & 87 & 9 & \\
\hline & P1018302000 & 2 & & & 0.30 & & & 0.04 & & & 96 & -2 & \\
\hline & P1018303000 & 2 & & & 0.18 & & & 0.03 & & & 61 & 9 & \\
\hline & P1018304000 & 2 & & & 0.31 & & & 0.02 & & & 99 & -7 & \\
\hline AKN564 & B0620101000 & 1 & 1.64 & 1.28 & 1.31 & 0.04 & 0.02 & 0.03 & 528 & 304 & 280 & -65 & 10 \\
\hline \multirow[t]{11}{*}{ IRAS F22456-5125 } & Z9073901000 & 1 & 0.06 & 0.32 & 0.28 & 0.04 & 0.06 & 0.06 & 42 & 110 & 105 & -787 & 11 \\
\hline & Z9073902000 & 1 & 0.07 & 0.19 & 0.17 & 0.01 & 0.01 & 0.01 & 81 & 64 & 69 & -803 & 22 \\
\hline & E8481401000 & 1 & 0.06 & 0.24 & 0.29 & 0.05 & 0.04 & 0.07 & 50 & 73 & 99 & -812 & 28 \\
\hline & Z9073901000 & 2 & 0.42 & & & 0.05 & & & 130 & & & -596 & \\
\hline & Z9073902000 & 2 & 0.35 & & & 0.01 & & & 97 & & & -612 & \\
\hline & $\mathrm{E} 8481401000$ & 2 & 0.44 & & & 0.06 & & & 110 & & & -617 & \\
\hline & Z9073901000 & 3 & 0.38 & 0.23 & 0.25 & 0.06 & 0.05 & 0.05 & 117 & 66 & 101 & -424 & 9 \\
\hline & Z9073902000 & 3 & 0.27 & 0.20 & 0.21 & 0.01 & 0.01 & 0.01 & 77 & 67 & 69 & -455 & 5 \\
\hline & E8481401000 & 3 & 0.33 & 0.38 & 0.26 & 0.05 & 0.06 & 0.06 & 96 & 151 & 94 & -447 & 13 \\
\hline & Z9073901000 & 4 & 0.09 & 0.17 & 0.10 & 0.05 & 0.05 & 0.04 & 56 & 66 & 42 & -311 & 3 \\
\hline & Z9073902000 & 4 & 0.08 & 0.21 & 0.14 & 0.01 & 0.01 & 0.01 & 40 & 63 & 66 & -323 & 5 \\
\hline
\end{tabular}


Table 3-Continued

\begin{tabular}{|c|c|c|c|c|c|c|c|c|c|c|c|c|c|}
\hline \multirow[t]{2}{*}{ Object } & \multirow[t]{2}{*}{ Obs ID } & \multirow[t]{2}{*}{ Comp } & \multicolumn{2}{|c|}{ Equivalent } & \multirow{2}{*}{$\begin{array}{c}\text { Width } \\
\text { O VIr } \\
(\AA)\end{array}$} & \multicolumn{3}{|c|}{$\sigma_{E W}$} & \multicolumn{3}{|c|}{ FWHM } & \multirow{2}{*}{$\begin{array}{c}\mathbf{V}_{r} \\
\left(\mathrm{~km} \mathrm{~s}^{-1}\right)\end{array}$} & \multirow{2}{*}{$\begin{array}{c}\sigma_{v e l} \\
\left(\mathrm{~km} \mathrm{~s}^{-1}\right)\end{array}$} \\
\hline & & & $\begin{array}{l}\mathrm{Ly} \beta \\
(\AA)\end{array}$ & $\begin{array}{l}\text { O VIb } \\
(\AA)\end{array}$ & & $\begin{array}{l}\operatorname{Ly} \beta \\
(\AA)\end{array}$ & $\begin{array}{c}\text { O VIb } \\
(\AA)\end{array}$ & $\begin{array}{l}\text { O VIr } \\
(\AA)\end{array}$ & $\operatorname{Ly} \beta$ & $\begin{array}{c}\mathrm{O} \text { VIb } \\
\left(\mathrm{km} \mathrm{s}^{-1}\right)\end{array}$ & O VIr & & \\
\hline \multirow{7}{*}{$\begin{array}{r}\text { MR 2251-178 } \\
\text { NGC7469 }\end{array}$} & E8481401000 & 4 & 0.12 & 0.18 & 0.14 & 0.05 & 0.05 & 0.04 & 35 & 105 & 39 & -327 & 1 \\
\hline & Z9073901000 & 5 & 0.70 & 0.51 & 0.54 & 0.08 & 0.06 & 0.07 & 190 & 142 & 143 & -118 & 19 \\
\hline & Z9073902000 & 5 & 0.68 & 0.67 & 0.46 & 0.01 & 0.01 & 0.01 & 182 & 136 & 134 & -135 & 22 \\
\hline & E8481401000 & 5 & 0.72 & 0.54 & 0.57 & 0.07 & 0.06 & 0.07 & 178 & 147 & 137 & -136 & 21 \\
\hline & P1111010000 & 1 & 1.62 & 0.70 & 0.40 & 0.03 & 0.01 & 0.01 & 418 & 234 & 155 & -300 & 23 \\
\hline & C0900101000 & 1 & & 0.40 & 0.30 & & 0.002 & 0.01 & & 151 & 137 & -1847 & 17 \\
\hline & C0900102000 & 1 & & 0.48 & 0.40 & & 0.01 & 0.01 & & 209 & 190 & -1878 & 3 \\
\hline
\end{tabular}


Table 4. Central Black Hole Masses

\begin{tabular}{|c|c|c|c|c|c|c|}
\hline Object & $\begin{array}{c}\text { FWHM H } \beta \\
\mathrm{km} \mathrm{s}^{-1}\end{array}$ & $\begin{array}{l}\text { Mass } \\
\times 10^{6}\end{array}$ & $\begin{array}{l}\log \lambda \mathrm{L}_{\lambda} \\
\operatorname{ergs~s}^{-1}\end{array}$ & $\begin{array}{l}\log \mathrm{L}_{B o l} \\
\operatorname{ergs~s}^{-1}\end{array}$ & $\mathrm{~L} / \mathrm{L}_{E d d}$ & reference $^{b}$ \\
\hline Mrk 79 & & 52.4 & 43.72 & 44.71 & 0.08 & 1 \\
\hline PG $0804+761$ & & 693 & 44.94 & 45.93 & 0.10 & 1 \\
\hline NGC 3516 & & 42.7 & 42.88 & 43.87 & 0.01 & 1 \\
\hline NGC 3783 & & 29.8 & 43.26 & 44.25 & 0.05 & 1 \\
\hline NGC 4051 & & 1.91 & 41.93 & 42.92 & 0.03 & 1 \\
\hline NGC 4151 & & 13.3 & 42.88 & 43.87 & 0.04 & 1 \\
\hline Mrk 279 & & 34.9 & 43.88 & 44.87 & 0.17 & 1 \\
\hline PG $1411+422$ & & 443 & 44.63 & 45.62 & 0.07 & 1 \\
\hline NGC5548 & & 67.1 & 43.51 & 44.50 & 0.04 & 1 \\
\hline Mrk 817 & & 49.4 & 43.82 & 44.81 & 0.10 & 1 \\
\hline Mrk 509 & & 143 & 44.28 & 45.27 & 0.10 & 1 \\
\hline NGC 7469 & & 12.2 & 43.72 & 44.71 & 0.33 & 1 \\
\hline Mrk 876 & & 279 & 44.98 & 45.97 & 0.27 & 1 \\
\hline Ton 951 & & 92.4 & 44.35 & 45.34 & 0.19 & 1 \\
\hline II Zw 136 & & 457 & 44.46 & 45.45 & 0.05 & 1 \\
\hline PG $1351+640$ & 1170 & 46 & 44.64 & 45.63 & 0.74 & 2 \\
\hline QSO $0045+3926$ & 5970 & 564.9 & 44.60 & 45.59 & 0.05 & 7 \\
\hline Ton S180 & 970 & 10.2 & 44.27 & 45.26 & 1.42 & 3 \\
\hline NGC 985 & 5670 & 202.8 & 43.80 & 44.79 & 0.02 & 3 \\
\hline IRAS F04250-5718 & 2580 & 144.0 & 44.87 & 45.86 & 0.40 & 3 \\
\hline Mrk 10 & 3050 & 25.6 & 43.08 & 44.07 & 0.04 & 4 \\
\hline IR $07546+3928$ & 2120 & 176.9 & $45.39^{a}$ & 46.39 & 1.10 & 8 \\
\hline Mrk 141 & 3600 & 65.0 & 43.60 & 44.59 & 0.05 & 3 \\
\hline RXJ 135515+561244 & 1100 & 9.4 & 43.98 & 44.97 & 0.79 & 3 \\
\hline PG $1404+226$ & 790 & 7.7 & 44.38 & 44.37 & 2.43 & 5 \\
\hline Mrk 290 & 5320 & 157.3 & 43.69 & 44.68 & 0.02 & 5 \\
\hline Akn 564 & 970 & 4.8 & 43.62 & 44.61 & 0.67 & 4 \\
\hline MR 2251-5125 & 3768 & 240.0 & 44.64 & 45.63 & 0.14 & 6 \\
\hline IRAS F22456-5125 & 3297 & 154.9 & $42.76^{a}$ & 45.53 & 0.16 & 6 \\
\hline WPVS 007 & 1502 & 11.5 & 43.59 & 44.58 & 0.27 & 6 \\
\hline RXJ 1230.8+0115 & & & & 45.0 & 0.13 & 9 \\
\hline
\end{tabular}

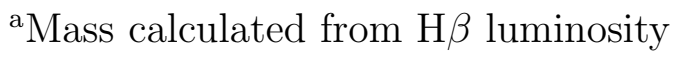

b1-Peterson et al. 2004, 2-Kaspi et al. 2000, 3-Grupe et al. 2004, 4-Botte et al. 2004, 
5-Vestergaard et al. 2006 6-This Paper, 7-Xu et al. 2003, 8-Marziani et al. 2003, 9-Ganguly et al. 2003

$$
{ }^{\mathrm{c}}{ }_{B o l}=9.8 \lambda \mathrm{L}_{5100}
$$

